\title{
Minimum-Energy State Estimation for Systems with Perspective Outputs
}

\author{
A. Pedro Aguiar, Member, IEEE, and João P. Hespanha, Senior Member, IEEE
}

\begin{abstract}
This paper addresses the state estimation of systems with perspective outputs. We derive a minimum-energy estimator which produces an estimate of the state that is "most compatible" with the dynamics, in the sense that it requires the least amount of noise energy to explain the measured outputs. Under suitable observability assumptions, the estimate converges globally asymptotically to the true value of the state in the absence of noise and disturbance. In the presence of noise, the estimate converges to a neighborhood of the true value of the state. These results are also extended to solve the estimation problem when the measured outputs are transmitted through a network. In that case, we assume that the measurements arrive at discrete-time instants, are time-delayed, noisy, and may not be complete. We show that the re-designed minimum-energy estimator preserves the same convergence properties. We apply these results to the estimation of position and orientation for a mobile robot that uses a monocular charged-coupled-device (CCD) camera mounted onboard to observe the apparent motion of stationary points. In the context of our application, the estimator can deal directly with the usual problems associated with vision systems such as noise, latency and intermittency of observations. Experimental results are presented and discussed.
\end{abstract}

Index Terms-Visual servo control, observers for nonlinear systems, estimation, robotics, networked control systems.

\section{INTRODUCTION}

$\mathbf{T}$ HE state estimation of nonlinear systems has received considerable attention in the literature during the last few decades. A particular branch of engineering where observers for nonlinear systems have been applied rather extensively is Computer Vision. In particular to solve the problems of pose estimation, shape tracking, 3D surface estimation, among others. In this paper, the practical motivation is the pose estimation problem for mobile robots using measurements from a charged-coupled-device (CCD) camera mounted onboard to observe the apparent motion of stationary points. The dynamics of these systems belong to the class of systems with perspective outputs, which will be introduced in the next subsection. The reader is referred to [1]-[3] for several other examples of perspective systems in the context of motion and shape estimation.

We are interested in designing state-estimators for systems with perspective outputs in the presence of noise and disturbance. We also consider the case when the measurements

This material is based upon work supported by the National Science Foundation under Grants No. ECS-0242798 and CCR-0311084. The work of A. Pedro Aguiar was supported by a Pos-Doc Fellowship PRAXIS XXI from the Portuguese Foundation of Science and Technology.

A. Pedro Aguiar and João P. Hespanha are with Center for Control Engineering and Computation, University of California, Santa Barbara, CA 93106-9560, USA. \{aguiar, hespanha\} @ece.ucsb.edu are transmitted through a network, arriving at discrete-time instants, possibly time-delayed, and incomplete.

The remainder of this section introduces the problem of state estimation for systems with perspective outputs using a minimum-energy approach, presents previous related work, describes the main contributions, and provides a brief overview of pose estimation of autonomous vehicles using visual information.

\section{A. State estimation for systems with perspective outputs}

Consider a continuous-time system described by

$$
\begin{aligned}
\dot{x} & =A(u) x+b(u)+G(u) \mathbf{d} \\
\alpha_{j} y_{j} & =C_{j}(u) x+d_{j}(u)+\mathbf{n}_{j} \quad j \in \mathcal{I}:=\{1,2, \ldots, N\}
\end{aligned}
$$

where ${ }^{1} A: \mathbb{R}^{m} \rightarrow \mathbb{R}^{n \times n}, b: \mathbb{R}^{m} \rightarrow \mathbb{R}^{n}, G: \mathbb{R}^{m} \rightarrow \mathbb{R}^{n \times n_{d}}$, $C_{j}: \mathbb{R}^{m} \rightarrow \mathbb{R}^{q_{j} \times n}, d_{j}: \mathbb{R}^{m} \rightarrow \mathbb{R}^{q_{j}}, x \in \mathbb{R}^{n}$ denotes the state of the system, $u \in \mathbb{R}^{m}$ its control input, $y_{j} \in \mathbb{R}^{q_{j}}$ its $j$ th perspective output, $\mathbf{d} \in \mathbb{R}^{n_{d}}$ an input disturbance that cannot be measured, and $\mathbf{n}_{j} \in \mathbb{R}^{q_{j}}$ measurement noise affecting the $j$ th output. We assume that the right-hand-side of (2) is always nonzero. The initial condition $x(0)$ of (1) and the signals $\mathbf{d}$ and $\mathbf{n}_{j}$ are all assumed deterministic but unknown. Each $\alpha_{j} \in \mathbb{R}$, $j \in \mathcal{I}$ denotes a scalar that is determined by a normalization constraint such as

$$
\left\|y_{j}\right\|=1 \quad \text { or } \quad v_{j}^{\prime} y_{j}=1
$$

where the $v_{j} \in \mathbb{R}^{q_{j}}$ denote constant vectors. We call (1)-(2) a state-affine system with multiple perspective outputs, or for short simply a system with perspective outputs. These type of systems are inspired by the (single output) perspective systems introduced by Ghosh et al. [1]. Notice that when the matrices $A, b$, and all the $C_{j}, d_{j}$ are constant, and $\mathbf{d}$ and all the $\mathbf{n}_{j}$ are zero, we essentially have a perspective linear system in the sense of [1].

In the last few years, the observability of perspective linear systems was been systematically studied in the literature. In [4], Dayawansa et al. provide an elegant algebraic observability test. For perspective linear systems without inputs it is never possible to recover the norm of the state because the system is homogeneous on the initial conditions. Therefore Dayawansa et al. [4] only consider state indistinguishability up a homogeneous scaling of the state. However, as shown in [5], in the presence of inputs it is in principle possible to recover the whole state from perspective outputs.

\footnotetext{
${ }^{1}$ To simplify the notation we use the short hand notation $A(u)$ to denote $A(u(t))$. The same applies for $b(\cdot), G(\cdot), C_{j}(\cdot)$, and $d_{j}(\cdot)$.
} 
Motivated by the above considerations, one of the main contributions of this paper is the design of a state-estimator for (1)-(2). In Section II we propose a minimum-energy estimator that produces an estimate for the state of the perspective system that is "most compatible" with the system's dynamics and measured outputs. In particular, the optimal state estimate $\hat{x}$ at time $t$ is defined to be the value for the state that is compatible with the observations collected up to time $t$, and the dynamics of the system for the "lowest" possible measurement noise and disturbance, with "lowest" understood in an integral-square sense. This formulation is purely deterministic but leads to a state-estimator that resembles a Kalman-Bucy filter. In fact, if the same approach towards state-estimation was applied to a linear system with linear outputs, one would arrive precisely at the Kalman-Bucy filter that would be obtained in a stochastic setting [6].

Minimum-energy estimators were first proposed by Mortensen [7] and further refined by Hijab [8]. Game theoretical versions of these estimators were proposed by McEneaney [9]. It was recently shown by Krener [10] that minimumenergy estimators are globally convergent when the system is observable for every input. Under less restrictive observability assumptions, in Section II-B we show that for perspective systems with multiple inputs, the state-estimator proposed has the desirable property that the state estimate converges asymptotically to the true value of the state in the absence of noise and disturbance. In the presence of noise, the estimate converges to a neighborhood of the true value of the state. We can therefore use this state-estimator to design output-feedback controllers by using the estimated state to drive state-feedback controllers.

Another problem that is tackled in this paper is state estimation for systems with perspective outputs when the measurements are transmitted through a network. Over the past few years there has been a considerable research effort in the area of networked control systems. The reader is refereed to [11] for a survey on this topic. In Section III we assume that the measurements are acquired only at discrete times $t_{i}^{\prime}$, $i=1,2, \ldots, k$, with $t_{1}^{\prime}<t_{2}^{\prime}<\ldots<t_{k}^{\prime}$, and that we only have access to them after a time-delay $\tau_{i}$. The sequence of measurements is therefore given by

$$
\mathbf{y}_{j}\left(t_{i}\right):=y_{j}\left(t_{i}^{\prime}\right)=y_{j}\left(t_{i}-\tau_{i}\right)
$$

where $\mathbf{y}$ denotes the time-delay observed variable, and $t_{i}:=$ $t_{i}^{\prime}+\tau_{i}$. We also suppose that the measurements may not be complete, that is, at time $t_{i}^{\prime}$ only the outputs $y_{j}$ with $j \in \mathcal{I}_{i}$ are available, where $\mathcal{I}_{i} \subseteq \mathcal{I}$, and the inclusion may be strict when some measurements are missing.

The problem under consideration is then to design an observer which estimates the continuous-time state vector $x(t)$ governed by equation (1), given the discrete time-delay measurements $\mathbf{y}\left(t_{i}\right)$ expressed by the output equation

$$
\begin{aligned}
\boldsymbol{\alpha}_{j_{i}} \mathbf{y}_{j}\left(t_{i}\right)= & C_{j}\left(u\left(t_{i}-\tau_{i}\right)\right) x\left(t_{i}-\tau_{i}\right) \\
& +d_{j}\left(u\left(t_{i}-\tau_{i}\right)\right)+\mathbf{n}_{j}\left(t_{i}-\tau_{i}\right) \quad j \in \mathcal{I}_{i}
\end{aligned}
$$

where $\boldsymbol{\alpha}_{j_{i}}$ is a normalization constraint such that (3) holds for $\mathbf{y}_{j}\left(t_{i}\right)$.
Convergence properties and observability conditions under which the estimate state $\hat{x}$ converges to the state $x$ are investigated in Section III-B.

\section{B. Pose estimation of autonomous vehicles using visual infor- mation}

A fundamental problem in mobile robotics is the determination of position and orientation with respect to an inertial coordinate system. A promising solution is to use a camera mounted on a robot to observe the apparent motion on the image of stationary points. The linear and angular velocities of the camera can be assumed known in its own coordinate system (possibly with errors due to noise) but not in the inertial coordinate system. This is quite reasonable in mobile robotics where the motion of the camera is determined by the applied control signals. The problem of estimating the position and orientation of a camera mounted on a rigid body from the apparent motion of point features has a long tradition in the computer vision literature (cf., e.g., [12]-[17] and references therein). Filtering-like or iterative algorithms that continuously improve the estimates as more data (i.e., images) are acquired, and that are robust with respect to measurement noise are especially desirable. Soatto et al. [14] formulated the visual motion estimation problem in terms of identification of nonlinear implicit systems with parameters on a topological manifold, and proposed a dynamic solution either in the local coordinates, or in the embedding space of the parameter manifold. In [17], rigid-body pose estimation using inertial sensors and a monocular camera is considered. A local convergent observer where the states evolve on $S O(3)$ is proposed (the rotation estimation is decoupled from the position estimation). In the area of wheeled mobile robots, Ma et al. [18] addressed the problem of tracking an arbitrarily shaped continuous ground curve by formulating it as controlling the shape of the curve in the image plane. Observability of the curve dynamics is studied and an extended Kalman filter is proposed to dynamically estimate the image quantities needed for feedback control from the actual noisy images. An application for landing an unmanned air vehicle using vision in the control loop is described in [19]. In [15], the autonomous aircraft landing problem based on measurements provided by airborne vision and inertial sensors is addressed. The authors cast the problem in a linear parametrically varying framework and solve it using tools that borrows from the theory of linear matrix inequalities. These results are extended in [20] to deal with the so-called out-of-frame events.

In Section IV we formulate the problem of estimating the position and orientation of a controlled rigid body using measurements from an on-board CCD camera as a state estimation problem of a perspective system. The problem is then solved by using the minimum-energy estimators derived in the previous sections. One of the main contributions is that, contrary to what happens with most previous algorithms, the ones proposed here are globally convergent provided that suitable observability assumptions are satisfied. These assumptions are independent of the initialization of the estimator and depend solely on the motion of the camera. 
Global convergence to the correct position and orientation is achieved in the absence of noise. When there is noise, the magnitude of the estimation error is essentially proportional to the amount of noise. Another difference with respect to several other algorithms is that we also estimate scale. This can be achieved either through known (scaling) information about the points observed, and/or through the knowledge of the camera's linear velocity. We also consider singular configurations for the points under observation, e.g., all points coplanar.

Another novelty of this paper is that we explicitly address the fact that the noisy measurements arrive at discrete-time instants, are time-delayed, and may not be complete. In this way, we can deal directly with the usual problems associated to vision systems such as noise, latency, and occlusions.

The theoretical results were experimentally validated by applying them to estimate the position and orientation of a mobile robot using measurements from an on-board CCD camera. We then use these estimates to close the feedback loop and control the robot to a desired position, defined with respect to visual landmarks. The results obtained are discussed in Section IV-C.

This paper builds upon and extends previous results by the authors [21]-[23].

\section{State Estimation for Systems with Perspective OUTPUTS}

Consider the perspective output system (1)-(2). For appropriate noise and disturbance signals, essentially every value for the state $x$ at a time $t \in \mathbb{R}$ is compatible with any outputs $y_{j}$ observed on the interval $[0, t)$. However, we will favor estimates for the state that can be made compatible with the measured outputs utilizing noise and disturbance with the lowest possible energy. In fact, we formulate state estimation as a deterministic optimization problem in which the estimate $\hat{x}(t)$ of the state at time $t \geq 0$ is the value for which the measured outputs can be made compatible with the system dynamics (1)-(2) for noise $\mathbf{n}_{j}$ and disturbance $\mathbf{d}$, with lowest integral-square-norm. More specifically, we address the following problem:

Problem 1: Given an input $u$ and measured outputs $y_{j}, j \in$ $\mathcal{I}$, defined on an interval $[0, t)$, compute the estimate $\hat{x}(t)$ of the state at time $t$ defined by

$$
\hat{x}(t):=\arg \min _{z \in \mathbb{R}^{n}} J(z, t)
$$

where

$$
\begin{gathered}
J(z ; t):=\min _{\mathbf{d}, \mathbf{n}_{j}, \alpha_{j}}\left\{\left(x(0)-\hat{x}_{0}\right)^{\prime} P_{0}\left(x(0)-\hat{x}_{0}\right)\right. \\
+\int_{0}^{t}\left(\|\mathbf{d}\|^{2}+\sum_{j=1}^{N}\left\|\mathbf{n}_{j}\right\|^{2}\right) d \tau: \\
x(t)=z, \dot{x}=A(u) x+b(u)+G(u) \mathbf{d}, \\
\left.\alpha_{j} y_{j}=C_{j}(u) x+d_{j}(u)+\mathbf{n}_{j}\right\}
\end{gathered}
$$

and $P_{0}>0, \hat{x}_{0}$ encode a-priori information about the state.

Remark 1: The approach just described towards state estimation can be viewed as the computation of a "generalized pseudo-inverse" that attempts to recover the current value of the state $x(t)$ from the measured output $y(\tau), \tau \in[0, t]$. To understand what is meant by this, let $U_{t}$ denote the triple consisting of the quantities that cannot be measured but affect the value of the state at time $t$. Namely, the initial state, past noise and past disturbances:

$$
U_{t}:=\{x(0) ; \mathbf{n}(\tau), \tau \in[0, t] ; \mathbf{d}(\tau), \tau \in[0, t]\} .
$$

We denote by $\mathcal{U}_{t}$ the space of such triples. The system dynamics (1)-(2) define the following two operators

$$
\begin{aligned}
X_{t}: \mathcal{U}_{t} & \rightarrow \mathbb{R}^{n} & O_{t}: \mathcal{U}_{t} & \rightarrow \mathcal{Y}_{t} \\
U_{t} & \mapsto x(t) & U_{t} & \mapsto Y_{t}
\end{aligned}
$$

where $\mathcal{Y}_{t}$ denotes the appropriate output space and $Y_{t}:=$ $\left\{y_{j}(\tau), \tau \in[0, t], j=1,2, \ldots, N\right\}$. One can then view state estimation as solving the following system of equations

$$
\hat{x}=X_{t}\left(\hat{U}_{t}\right) \quad Y_{t}=O_{t}\left(\hat{U}_{t}\right)
$$

for the unknowns $\hat{x}$ and $\hat{U}_{t}$. If the observation operator $O_{t}$ had a left inverse $O_{t}^{-1}$ (e.g., in the absence of noise and if the system was observable) the solution to (7) would be unique and given by $\hat{x}=X_{t}\left(O_{t}^{-1}\left(Y_{t}\right)\right)$. However, in general this is not the case and the approach that we propose is to replace the left inverse of $O_{t}$ by its "pseudo-inverse." In particular, we define the estimate to be $\hat{x}=X_{t}\left(O_{t}^{\perp}\left(Y_{t}\right)\right)$, where $O_{t}^{\perp}\left(Y_{t}\right)$ denotes the min-norm solution to $Y_{t}=O_{t}\left(U_{t}\right)$, i.e., $O_{t}^{\perp}\left(Y_{t}\right)=$ $\arg \min _{\substack{\left.U_{t} \in \mathcal{U}_{t}: \\ U_{t}\right)}}\left\|U_{t}\right\|_{\mathcal{U}_{t}}$. The norm $\|\cdot\|_{\mathcal{U}_{t}}$ (or more precisely its square) is specified by the cost (6).

Remark 2: In general, for nonlinear systems, the approach proposed here to construct an estimator leads to an infinite dimensional observer with state $J(\cdot, t)$ evolving according to a first-order nonlinear partial differential equation of HamiltonJacobi type, driven by the observations. The negative of $J(z, t)$ defined by (6) is the information state introduced in [24], [25] and can be interpreted as a measure of the likelihood of state $x=z$ at time $t$.

\section{A. The observer equations}

We now present the observer equations that can be derived using dynamic programming. The following result solves Problem 1.

Theorem 1: The solution to the state estimation problem defined by (5) and (6) is given by

$$
\begin{aligned}
\dot{Q} & =A(u) Q+Q A(u)^{\prime}+G(u) G(u)^{\prime}-Q W Q \\
\dot{\hat{x}} & =(A(u)-Q W) \hat{x}+b(u)-Q w
\end{aligned}
$$

with $Q(0)=P_{0}^{-1}, \hat{x}(0)=\hat{x}_{0}$, and

$$
\begin{aligned}
W(t) & :=\sum_{j=1}^{N} C_{j}^{\prime}(u)\left(I-\frac{y_{j} y_{j}^{\prime}}{\left\|y_{j}\right\|^{2}}\right) C_{j}(u) \\
w(t) & :=\sum_{j=1}^{N} C_{j}^{\prime}(u)\left(I-\frac{y_{j} y_{j}^{\prime}}{\left\|y_{j}\right\|^{2}}\right) d_{j}(u) \quad t \geq 0 .
\end{aligned}
$$


Furthermore, the cost function $J(z ; t)$ defined in (6) is quadratic and can be written as

$$
J(z ; t)=(z-\hat{x}(t))^{\prime} P(t)(z-\hat{x}(t))+c(t)
$$

where $c(t)$ satisfies

$$
\dot{c}=\hat{x}^{\prime} W \hat{x}+\sum_{j=1}^{N} d_{j}^{\prime}\left(I-\frac{y_{j} y_{j}^{\prime}}{\left\|y_{j}\right\|^{2}}\right) d_{j} \quad c(0)=0 .
$$

Before proving Theorem 1 note that we can rewrite the state estimation equation (9) as

$\dot{\hat{x}}=A(u) \hat{x}+b(u)+Q \sum_{j=1}^{N} C_{j}(u)^{\prime}\left(\hat{\alpha}_{j} y_{j}-C_{j}(u) \hat{x}-d_{j}(u)\right)$

$\hat{\alpha}_{j}:=y_{j}^{\prime}\left(C_{j}(u) \hat{x}+d_{j}(u)\right) /\left\|y_{j}\right\|^{2}$, which emphasizes the parallel between (9) and a Kalman-Bucy filter for linear systems. In fact, if we apply the same approach for linear systems, the resulting minimum-energy estimate is identical to that generated by the Kalman-Bucy filter for the analogous linear stochastic model with initial covariance $P_{0}^{-1}$. In that case, $P^{-1}(t)$ is the covariance matrix of the estimation error at time $t$.

Proof: The function $J(z ; t), z \in \mathbb{R}^{n}, t \geq 0$ defined in (6) can be viewed as a cost-to-go. Computing it using dynamic programming, and completing the squares, we get

$$
\begin{aligned}
J_{t}(z ; t)= & \min _{\mathbf{d}, \alpha_{j}}\|\mathbf{d}\|^{2}+\sum_{j=1}^{N}\left\|\alpha_{j} y_{j}-C_{j} z-d_{j}\right\|^{2} \\
& -J_{z}(z ; t)(A z+b+G \mathbf{d}) \\
= & -\frac{1}{4}\left\|G^{\prime} J_{z}(z ; t)^{\prime}\right\|^{2}-J_{z}(z ; t)(A z+b) \\
& +\sum_{j=1}^{N}\left(C_{j} z+d_{j}\right)^{\prime}\left(I-\frac{y_{j} y_{j}^{\prime}}{\left\|y_{j}\right\|^{2}}\right)\left(C_{j} z+d_{j}\right)
\end{aligned}
$$

where $J_{t}$ and $J_{z}$ denote the partial derivatives of $J$ with respect to $t$ and $z$, respectively. To simplify the notation, we have suppressed the dependence on $u$ of the matrices $A, b, G, C_{j}$ and $d_{j}$. The value of $J(z ; t)$ can be determined from the linear partial differential equation (12) with initial condition

$$
J(z ; 0)=\left(z-\hat{x}_{0}\right)^{\prime} P_{0}\left(z-\hat{x}_{0}\right) \quad \forall z \in \mathbb{R}^{n} .
$$

It turns out that the solution to (12)-(13) can be written as (10) for appropriately defined signals $\hat{x}(t)$ and $c(t)$. The signal $\hat{x}$ is then precisely the estimate for the state $x$ of the perspective linear system. Moreover, matching (13) with (10) we conclude that $P(0)=P_{0}, \hat{x}(0)=\hat{x}_{0}$, and $c(0)=0$. To verify that the solution to (12)-(13) can indeed be written as (10), we substitute it into (12) and obtain

$$
\begin{aligned}
& z^{\prime}\left(\dot{P}+P A+A^{\prime} P+P G G^{\prime} P-W\right) z \\
& +2 z^{\prime}\left(-P \dot{\hat{x}}-\dot{P} \hat{x}-P G G^{\prime} P \hat{x}-A^{\prime} P \hat{x}+P b-w\right) \\
& +\dot{c}+2 \hat{x}^{\prime} P \dot{\hat{x}}+\hat{x}^{\prime} \dot{P} \hat{x}+\hat{x}^{\prime} P G G^{\prime} P \hat{x}-2 \hat{x}^{\prime} P b \\
& -\sum_{j=1}^{N} d_{j}^{\prime}\left(I-\frac{y_{j} y_{j}^{\prime}}{\left\|y_{j}\right\|^{2}}\right) d_{j}=0 \quad \forall z \in \mathbb{R}^{n} .
\end{aligned}
$$

For this quadratic law in $z$ to be identically equal to zero the following should hold:

$$
\begin{array}{r}
\dot{P}+P A+A^{\prime} P+P G G^{\prime} P-W=0 \\
-P \dot{\hat{x}}-\dot{P} \hat{x}-P G G^{\prime} P \hat{x}-A^{\prime} P \hat{x}+P b-w=0 \\
\dot{c}+2 \hat{x}^{\prime} P \dot{\hat{x}}+\hat{x}^{\prime} \dot{P} \hat{x}+\hat{x}^{\prime} P G G^{\prime} P \hat{x}-2 \hat{x}^{\prime} P b \\
-\sum_{j=1}^{N} d_{j}^{\prime}\left(I-\frac{y_{j} y_{j}^{\prime}}{\left\|y_{j}\right\|^{2}}\right) d_{j}=0 .
\end{array}
$$

Substituting (14) in (15) and these two equations in (16), we obtain (11) and

$$
\begin{aligned}
-\dot{P} & =P A+A^{\prime} P+P G G^{\prime} P-W \\
P \dot{\hat{x}} & =P A \hat{x}+P b-W \hat{x}-w
\end{aligned}
$$

It turns out that $P(t)$ remains positive definite for all time (see Claim 1 in Appendix). Therefore, (18) is actually equivalent to (9) with $Q=P^{-1}$. Using the fact that $\dot{Q}=-Q \dot{P} Q$, it is straightforward to conclude that the matrix $Q$ can be generated directly from (8).

\section{B. Estimator convergence}

We are now interested in determining under which conditions the state estimate $\hat{x}$ converges to the true state $x$ of the perspective system. The following technical assumption is needed:

Assumption 1: There exist positive constants $\delta, \Delta$ such that $\delta I \leq G(u) G^{\prime}(u) \leq \Delta I, \forall u \in \mathbb{R}^{m}$.

This assumption essentially guarantees that $G(u)$ is bounded and full-row rank, "uniformly" over all possible inputs. This type of assumption is typically used in minimum-energy estimators [9]. It can generally be replaced by a controllability assumption of (1) through the input $\mathbf{d}$. This controllability condition also arises in Kalman filtering to guarantee a positive definite solution to the algebraic Riccati equation. However, we do not pursue this case because, in practice, $G$ is a tuning parameter of the estimator that can be chosen by the designer. The following result establishes the convergence of the state estimate.

Theorem 2: Assuming that the solution to the perspective system (1)-(2) exists globally, the solution to state estimator (8)-(9) also exists globally. Moreover, when Assumption 1 holds and $Q$ remains uniformly bounded, there exist a constant $c>0$ possibly dependent on $P_{0}$, and positive constants $\lambda, \gamma_{d}, \gamma_{1}, \ldots, \gamma_{N}$ such that

$$
\begin{aligned}
\|\tilde{x}(t)\| \leq & c e^{-\lambda t}\|\tilde{x}(0)\|+\gamma_{d} \sup _{\tau \in(0, t)}\|\mathbf{d}(\tau)\| \\
& +\sum_{j=1}^{N} \gamma_{j} \sup _{\tau \in(0, t)}\left\|\mathbf{n}_{j}(\tau)\right\| \quad t \geq 0
\end{aligned}
$$

where $\tilde{x}(t):=\hat{x}(t)-x(t)$ denotes the state estimation error.

Proof: See the Appendix.

Remark 3: If the noise and input disturbances are $\mathcal{L}_{2}$ signals, then one can even prove convergence of the estimation error to zero. This was done in [10] for certain classes of systems that are uniformly observable for every input. 
Some assumption on the observability ${ }^{2}$ of (1)-(2) would be expected to achieve convergence of the state estimate $\hat{x}$ to the state $x$. In Theorem 2 this assumption appears in the form of the requirement that $Q$ remains uniformly bounded. This assumption is less restrictive than the usual one of requiring the system to be uniformly observable for every input [10], which generally does not happen for perspective systems. In the rest of this section we investigate conditions under which $Q$ remains uniformly bounded.

From (8) it is clear that $Q$ remains bounded if $W(t) \geq \epsilon I>$ $0, \forall t \geq 0$ because in this case the term $-Q W Q$ eventually dominates for very large $Q$. However, this case is not very interesting because, e.g., for the single output case $(N=1)$ the matrix $W$ typically has rank equal to $\left(\operatorname{rank} C_{1}\right)-1 \leq$ $n-1$. The following Lemma provides a significantly weaker condition for the boundedness of $Q$.

Lemma 1: For a given input $u, \mathbf{d}, \mathbf{n}_{j}, j \in\{1, \ldots, N\}$ the matrix $Q$ remains bounded along trajectories of the system (1)-(2) and state-estimator (8)-(9), provided that there exist positive constants $T, \epsilon$ such that the following condition

$$
\frac{1}{T} \int_{0}^{T} \Phi(t+\tau, t)^{\prime} W(t+\tau) \Phi(t+\tau, t) d \tau \geq \epsilon I>0
$$

$\forall t \geq 0$ holds, where $\Phi(t, \tau)$ denotes the state transition matrix of $\dot{z}=A(u) z$.

Proof: See the Appendix.

To get some intuition for the meaning of (20) note that for $\int_{0}^{T} \Phi(t+\tau, t)^{\prime} W(t+\tau) \Phi(t+\tau, t) d \tau$ to be singular, there would have to be a vector $x_{0}$ such that

$x_{0}^{\prime} \Phi(t+\tau, t)^{\prime} W(t+\tau) \Phi(t+\tau, t) x_{0}=0, \forall \tau \in(0, T), t \geq 0$.

In that case, using the definition of $W$ and the fact that $W(t+\tau) \geq 0$ we conclude that

$$
\left(I-\frac{y_{j}(t+\tau) y_{j}(t+\tau)^{\prime}}{\left\|y_{j}(t+\tau)\right\|^{2}}\right) C_{j}(u(t+\tau)) \Phi(t+\tau, t) x_{0}=0
$$

or equivalently,

$$
\beta_{j}(t+\tau) y_{j}(t+\tau)=C_{j}(u(t+\tau)) \Phi(t+\tau, t) x_{0}
$$

$\forall \tau \in(0, T), t \geq 0, j \in\{1, \ldots, N\}$, for appropriate scalars $\beta_{j}(t)$. In essence this means that (20) fails when all the $y_{j}$ evolve as if $b(u)$, d, and all the $d_{j}(u)$ and $\mathbf{n}_{j}$ were zero. In fact, we can view (20) as a persistence of excitation-like condition that requires $x$ to evolve is some interesting way, other than just following the homogeneous dynamics of (1) (2), along which scaling information could not be recovered.

It is interesting to note the parallel between the integral in (20) and the constructibility Gramian for linear system [26, Section 3.3]. In fact, if $W$ were replaced by $\sum_{j=1}^{N} C_{j}^{\prime} C_{j}$, the integral in (20) is precisely the constructibility Gramian for the system (1) with linear outputs $C_{j} x+d_{j}+\mathbf{n}_{j}, j \in$ $\{1,2, \ldots, N\}$.

Combining Theorem 2 and Lemma 1 we obtain the following:

\footnotetext{
${ }^{2}$ In the present setup, the correct notion is actually constructability because we are attempting to reconstruct the state from past outputs [26, Section 3.3].
}

Corollary 1: When Assumption 1 holds and there exist constants $T, \epsilon$ such that (20) holds, the state estimate $\hat{x}$ converges exponentially fast to the state $x$ in the absence of disturbance input and measurement noise. When the disturbance and noise are bounded but nonzero, $\hat{x}$ converges to a neighborhood of the true state $x$.

Remark 4: Given the input-to-state stability (ISS) like result in Theorem 2, we can use the state-estimator to design outputfeedback controllers by using the state estimate $\hat{x}$ to drive state-feedback controllers. This is possible when the statefeedback controllers are robust (in ISS sense) with respect to state perturbations (cf., e.g., [27], [28], [29, Section 5.3]) and (20) holds. However, in general condition (20) may be difficult to check since it depends on the control input $u$. The investigation of a relaxed condition of persistence of excitation such as the one introduced in [30] is a topic of current research.

\section{STATE Estimation FROM Discrete Noisy Time-Delayed Measurements}

This section addresses the state estimation of continuoustime systems with perspective outputs, whose measurements arrive at discrete-time instants, are time-delayed, noisy, and may not be complete.

Consider the perspective system described by the state equation (1) and the output equation (4). From (1) we conclude that $x\left(t_{i}\right)$ satisfies

$$
\begin{aligned}
x\left(t_{i}\right)= & \Phi\left(t_{i}, t_{i}-\tau_{i}\right) x\left(t_{i}-\tau_{i}\right) \\
& +\int_{t_{i}-\tau_{i}}^{t_{i}} \Phi\left(t_{i}, \sigma\right)[b(u(\sigma))+G(u(\sigma)) \mathbf{d}(\sigma)] d \sigma
\end{aligned}
$$

where $\Phi\left(t, t_{0}\right)$ is the transition matrix satisfying the differential equation $\dot{\Phi}=A(u) \Phi$. Therefore,

$$
\begin{aligned}
x\left(t_{i}-\tau_{i}\right)= & \Phi^{-1}\left(t_{i}, t_{i}-\tau_{i}\right) x\left(t_{i}\right)-\Phi^{-1}\left(t_{i}, t_{i}-\tau_{i}\right) \times \\
& \int_{t_{i}-\tau_{i}}^{t_{i}} \Phi\left(t_{i}, \sigma\right)[b(u(\sigma))+G(u(\sigma)) \mathbf{d}(\sigma)] d \sigma .
\end{aligned}
$$

Substituting this equation in (4) we get

$$
\boldsymbol{\alpha}_{j_{i}} \mathbf{y}_{j}\left(t_{i}\right)=\bar{C}_{j}(u) x\left(t_{i}\right)+\bar{d}_{j}(u)+\overline{\mathbf{n}}_{j}\left(t_{i}\right) \quad j \in \mathcal{I}_{i}
$$

where

$$
\begin{aligned}
\bar{C}_{j}(u) & :=C_{j}\left(u\left(t_{i}-\tau_{i}\right)\right) \Phi\left(t_{i}-\tau_{i}, t_{i}\right) \\
\bar{d}_{j}(u) & :=-\bar{C}_{j}(u) \int_{t_{i}-\tau_{i}}^{t_{i}} \Phi\left(t_{i}, \sigma\right) b(u(\sigma)) d \sigma+d_{j}\left(u\left(t_{i}-\tau_{i}\right)\right) \\
\overline{\mathbf{n}}_{j}\left(t_{i}\right) & :=-\bar{C}_{j}(u) \int_{t_{i}-\tau_{i}}^{t_{i}} \Phi\left(t_{i}, \sigma\right) G(u(\sigma)) \mathbf{d}(\sigma) d \sigma+\mathbf{n}_{j}\left(t_{i}-\tau_{i}\right) .
\end{aligned}
$$

The minimum-energy estimation problem can now be stated as follows:

Problem 2: Given an input $u$ defined on an interval $[0, t)$, and measured outputs $\mathbf{y}_{j}\left(t_{i}\right), j \in \mathcal{I}_{i}$ with $i=0,1, \ldots k, t_{0}:=$ $0 \leq t_{1} \leq \cdots \leq t_{k} \leq t$, compute the estimate $\hat{x}(t)$ of the state at time $t$ defined by

$$
\hat{x}(t):=\arg \min _{z \in \mathbb{R}^{n}} J(z, t)
$$


where

$$
\begin{gathered}
J(z ; t):=\min _{\substack{\mathbf{d}:[0, t), \overline{\mathbf{n}}_{j}\left(t_{i}\right), \boldsymbol{\alpha}_{j_{i}} \\
i=0,1, \ldots k}}\left\{\left(x(0)-\hat{x}_{0}\right)^{\prime} P_{0}\left(x(0)-\hat{x}_{0}\right)\right. \\
+\int_{0}^{t}\|\mathbf{d}(\sigma)\|^{2} d \sigma+\sum_{i=0}^{k} \sum_{j \in \mathcal{I}_{i}}\left\|\overline{\mathbf{n}}_{j}\left(t_{i}\right)\right\|^{2}: \\
x(t)=z, \dot{x}=A(u) x+b(u)+G(u) \mathbf{d}, \\
\left.\boldsymbol{\alpha}_{j_{i}} \mathbf{y}_{j}\left(t_{i}\right)=\bar{C}_{j}(u) x\left(t_{i}\right)+\bar{d}_{j}(u)+\overline{\mathbf{n}}_{j}\left(t_{i}\right)\right\}
\end{gathered}
$$

and $P_{0}>0, \hat{x}_{0}$ encode a-priori information about the state.

The state estimate $\hat{x}(t)$ can be interpreted as the value for which the measured outputs can be made compatible with the system dynamics (1) and (22) utilizing noise $\overline{\mathbf{n}}_{j}$ and input disturbance $\mathbf{d}$ with the lowest possible energy. This formulation considers the case when all the measurements may not be available at each time $t_{i}$ because $\mathcal{I}_{i}$ can be a strict subset of $\mathcal{I}$.

\section{A. The observer equations}

In what follows, given a signal $x$ with a discontinuity at time $t$, we denote by $x\left(t^{-}\right)$the limit from below of $x(\tau)$ as $\tau \uparrow t$, i.e., $x\left(t^{-}\right):=\lim _{\tau \uparrow t} x(\tau)$. Without loss of generality we take all signals to be continuous from above at every point, i.e., $x(t)=\lim _{\tau \downarrow t} x(\tau)$. The following result solves Problem 2.

Theorem 3: The state estimate $\hat{x}(t)$ at time $t \geq t_{0}:=0$ defined by (23) and (24) can be computed as a solution to the impulse system:

- for $t_{i} \leq t<t_{i+1}, i=0,1, \ldots, k$

$$
\begin{aligned}
\dot{P}(t)= & -P(t) A(u)-A(u)^{\prime} P(t) & & \\
& -P(t) G(u) G(u)^{\prime} P(t) & & P\left(t_{i}\right)=P_{i} \\
\dot{\hat{x}}(t)= & A(u) \hat{x}(t)+b(u) & & \hat{x}\left(t_{i}\right)=\hat{x}_{i}
\end{aligned}
$$

- at $t=t_{i+1}, i=0,1, \ldots, k-1$

$$
\begin{aligned}
P\left(t_{i+1}\right)= & P\left(t_{i+1}^{-}\right)+W\left(t_{i+1}\right) \\
\hat{x}\left(t_{i+1}\right)= & \hat{x}\left(t_{i+1}^{-}\right) \\
& -P\left(t_{i+1}\right)^{-1}\left[W\left(t_{i+1}\right) \hat{x}\left(t_{i+1}^{-}\right)+w\left(t_{i+1}\right)\right]
\end{aligned}
$$

where

$$
\begin{aligned}
W\left(t_{i+1}\right) & :=\sum_{j \in \mathcal{I}_{i+1}} \bar{C}_{j}^{\prime}(u)\left(I-\frac{\mathbf{y}_{j}\left(t_{i+1}\right) \mathbf{y}_{j}\left(t_{i+1}\right)^{\prime}}{\left\|\mathbf{y}_{j}\left(t_{i+1}\right)\right\|^{2}}\right) \bar{C}_{j}(u) \\
w\left(t_{i+1}\right) & :=\sum_{j \in \mathcal{I}_{i+1}} \bar{C}_{j}^{\prime}(u)\left(I-\frac{\mathbf{y}_{j}\left(t_{i+1}\right) \mathbf{y}_{j}\left(t_{i+1}\right)^{\prime}}{\left\|\mathbf{y}_{j}\left(t_{i+1}\right)\right\|^{2}}\right) \bar{d}_{j}(u)
\end{aligned}
$$

Furthermore, the cost function $J(z ; t)$ defined in (24) is quadratic and can be written as

$$
J(z ; t)=(z-\hat{x}(t))^{\prime} P(t)(z-\hat{x}(t))+c(t)
$$

where $c(0)=0$ and, for all $i=0,1, \ldots k-1$,

$$
\begin{aligned}
c(t)= & c\left(t_{i}\right), \quad t_{i} \leq t<t_{i+1} \\
c(t)= & -\left(P\left(t^{-}\right) \hat{x}\left(t^{-}\right)+\hat{x}\left(t^{-}\right)^{\prime} P\left(t^{-}\right) x\left(t^{-}\right)+c\left(t^{-}\right)\right. \\
& -w(t))^{\prime}\left[P\left(t^{-}\right)+W(t)\right]^{-T}\left(P\left(t^{-}\right) \hat{x}\left(t^{-}\right)-w(t)\right) \\
& +\sum_{j \in \mathcal{I}_{i+1}} \bar{d}_{j}\left(I-\frac{\mathbf{y}_{j} \mathbf{y}_{j}^{\prime}}{\left\|\mathbf{y}_{j}\right\|^{2}}\right) \bar{d}_{j}, \quad t=t_{i+1}
\end{aligned}
$$

Proof: We start by proving (30). Take some $t \in\left(t_{i}, t_{i+1}\right)$. Since $J(z ; t)$ is a cost-to-go it must satisfy the dynamic programming equation

$$
J_{t}(z ; t)=-\frac{1}{4}\left\|G^{\prime} J_{z}(z ; t)^{\prime}\right\|^{2}-J_{z}(z ; t)(A z+b)
$$

where $J_{t}$ and $J_{z}$ denote the partial derivatives of $J$ with respect to $t$ and $z$, respectively. For $k=0$, the value of $J(z ; t)$ is determined from the linear partial differential equation (33) with initial condition

$$
J(z ; 0)=\left(z-\hat{x}_{0}\right)^{\prime} P_{0}\left(z-\hat{x}_{0}\right) \quad \forall z \in \mathbb{R}^{n}
$$

and can be written as (30) for appropriately defined signals $\hat{x}(t)$ and $c(t)$. The signal $\hat{x}$ is then precisely the estimate for the state $x$ of the perspective system (1), (22). Moreover, matching (34) with (30) we conclude that $P(0)=P_{0}, \hat{x}(0)=\hat{x}_{0}, c(0)=$ 0 . To verify that the solution to (33)-(34) can be written as (30), we substitute this equation in (33), and obtain

$$
\begin{aligned}
& z^{\prime}\left(\dot{P}+P A+A^{\prime} P+P G G^{\prime} P\right) z \\
& \quad+2 z^{\prime}\left(-P \dot{\hat{x}}-\dot{P} \hat{x}-P G G^{\prime} P \hat{x}-A^{\prime} P \hat{x}+P b\right) \\
& +\dot{c}+2 \hat{x}^{\prime} P \dot{\hat{x}}+\hat{x}^{\prime} \dot{P} \hat{x}+\hat{x}^{\prime} P G G^{\prime} \hat{x}-2 \hat{x}^{\prime} P b=0 \quad \forall z \in \mathbb{R}^{n} .
\end{aligned}
$$

This equation holds provided that

$$
\begin{aligned}
\dot{P}+P A+A^{\prime} P+P G G^{\prime} P & =0, \\
-P \dot{\hat{x}}-\dot{P} \hat{x}-P G G^{\prime} P \hat{x}-A^{\prime} P \hat{x}+P b & =0, \\
\dot{c}+2 \hat{x}^{\prime} P \dot{\hat{x}}+\hat{x}^{\prime} \dot{P} \hat{x}+\hat{x}^{\prime} P G G^{\prime} \hat{x}-2 \hat{x}^{\prime} P b & =0 .
\end{aligned}
$$

Substituting (35) in (36) and these two equations in (37), we conclude that (25)-(26) and (30) hold for $0 \leq t<t_{1}$. Notice also that $P(t)$ remains positive definite for all $0 \leq t<t_{1}$ (see Claim 1 in Appendix, but using (25) instead of (17)).

Consider now the case $t=t_{k}, k>0$. From (24), we notice that $J\left(z ; t_{k}\right)$ can be written as

$$
\begin{aligned}
& J\left(z ; t_{k}\right)=\min _{\boldsymbol{\alpha}_{j_{k}}}\left\{\operatorname { m i n } _ { \substack { \mathbf { d } : [ 0 , t _ { k } ) , \boldsymbol { \alpha } _ { j _ { i } } \\
i = 0 , 1 , \ldots k - 1 } } \left\{\left(x(0)-\hat{x}_{0}\right)^{\prime} P_{0}\left(x(0)-\hat{x}_{0}\right)\right.\right. \\
& \quad+\int_{0}^{t_{k}}\|\mathbf{d}(\sigma)\|^{2} d \sigma+\sum_{j \in \mathcal{I}_{k}}\left\|\boldsymbol{\alpha}_{j_{k}} \mathbf{y}_{j}\left(t_{k}\right)-\bar{C}_{j} x\left(t_{k}\right)-\bar{d}_{j}\right\|^{2} \\
& \quad+\sum_{i=0}^{k-1} \sum_{j \in \mathcal{I}_{i}}\left\|\boldsymbol{\alpha}_{j_{i}} \mathbf{y}_{j}\left(t_{i}\right)-\bar{C}_{j} x\left(t_{i}\right)-\bar{d}_{j}\right\|^{2}: \\
& \left.\left.\quad x\left(t_{k}^{-}\right)=x\left(t_{k}\right)=z, \dot{x}=A x+b+G \mathbf{d}\right\}\right\} \\
& =\min _{\boldsymbol{\alpha}_{j_{k}}}\left\{J\left(z ; t_{k}^{-}\right)+\sum_{j \in \mathcal{I}_{k}}\left\|\boldsymbol{\alpha}_{j_{k}} \mathbf{y}_{j}\left(t_{k}\right)-\bar{C}_{j} x\left(t_{k}\right)-\bar{d}_{j}\right\|^{2}\right\}
\end{aligned}
$$

For $k=1$ we already have shown that $J\left(z, t_{1}^{-}\right)$is given by (30). Assuming that it has the same form at time $t_{1}$, 
substituting it in the left and right-hand-side of (38), after some algebraic manipulation, we obtain

$$
\begin{aligned}
& z^{\prime}\left[P\left(t_{k}\right)-P\left(t_{k}^{-}\right)-W\left(t_{k}\right)\right] z \\
& +2 z^{\prime}\left[-P\left(t_{k}\right) \hat{x}\left(t_{k}\right)+P\left(t_{k}^{-}\right) \hat{x}\left(t_{k}^{-}\right)-w\left(t_{k}\right)\right] \\
& \quad+c\left(t_{k}\right)+\hat{x}\left(t_{k}\right)^{\prime} P\left(t_{k}\right) x\left(t_{k}\right)-\hat{x}\left(t_{k}^{-}\right)^{\prime} P\left(t_{k}^{-}\right) x\left(t_{k}^{-}\right) \\
& \quad-c\left(t_{k}^{-}\right)-\sum_{j \in \mathcal{I}_{k}} \bar{d}_{j}\left(I-\frac{\mathbf{y}_{j} \mathbf{y}_{j}^{\prime}}{\left\|\mathbf{y}_{j}\right\|^{2}}\right) \bar{d}_{j}=0
\end{aligned}
$$

where the definitions in (29) were used. This equation holds for $k=1$ provided that

$$
\begin{array}{r}
P\left(t_{k}\right)-P\left(t_{k}^{-}\right)-W\left(t_{k}\right)=0 \\
-P\left(t_{k}\right) \hat{x}\left(t_{k}\right)+P\left(t_{k}^{-}\right) \hat{x}\left(t_{k}^{-}\right)-w\left(t_{k}\right)=0 \\
c\left(t_{k}\right)+\hat{x}\left(t_{k}\right)^{\prime} P\left(t_{k}\right) x\left(t_{k}\right)-\hat{x}\left(t_{k}^{-}\right)^{\prime} P\left(t_{k}^{-}\right) x\left(t_{k}^{-}\right) \\
-c\left(t_{k}^{-}\right)-\sum_{j \in \mathcal{I}_{k}} \bar{d}_{j}\left(I-\frac{\mathbf{y}_{j} \mathbf{y}_{j}^{\prime}}{\left\|\mathbf{y}_{j}\right\|^{2}}\right) \bar{d}_{j}=0 .
\end{array}
$$

Thus, substituting (39) in (40) and these two equations in (41), we conclude that (27)-(28), and (32) hold.

Notice that $P_{1}:=P\left(t_{1}\right)=P\left(t_{1}^{-}\right)+W\left(t_{1}\right)$ is positive definite because $P\left(t_{1}^{-}\right)>0$ as it was proved above, and $W\left(t_{i}\right) \geq 0, i=1, \ldots, k$. Therefore, substituting the initial condition (34) by

$$
J\left(z ; t_{1}\right)=\left(z-\hat{x}_{1}\right)^{\prime} P_{1}\left(z-\hat{x}_{1}\right) \quad z \in \mathbb{R}^{n}
$$

with $\hat{x}_{1}=\hat{x}\left(t_{1}\right)$, and solving the linear partial differential equation (33), it follows that (25)-(27) hold for $0 \leq t<t_{2}$. Applying this line of reasoning successively until $i=k$ we conclude (30) and that $\hat{x}(t)$ given by (25)-(28) is indeed the solution to Problem 2.

\section{B. Estimator convergence}

In this section we investigate under what conditions the estimate $\hat{x}$ provided by Theorem 3 converges to the true state $x$ of the perspective system. In addition to Assumption 1, the following technical assumption is needed:

Assumption 2: Let $\operatorname{Num}(t, \sigma), 0 \leq \sigma<t$ denotes the number of time instants at which measurement arrive in the open interval $(\sigma, t)$. There exist positive constants $\tau_{D}$ and $N_{0}$, for which the following condition holds:

$$
\operatorname{Num}(t, \sigma) \leq N_{0}+\frac{t-\sigma}{\tau_{D}}
$$

The constant $\tau_{D}$ is called the average dwell-time and $N_{0}$ the chatter bound.

In a broad sense, this assumption is used to restrict the average interval between consecutive arrival of measurements to be no less than $\tau_{D}$. This type of condition typically arises in the context of logic-based switching control (cf., e.g., [31] and references therein). In our context it guarantees that the summation in (24) will not grow unbounded due to "too frequent" measurements. This assumption is purely technical and is only used to simplify the analysis. Moreover, in practice it always holds.

The following result establishes the convergence of the state estimate.
Theorem 4: Assuming that the solution to the process (1), (22) exists globally, the solution to the impulse state estimator (25)-(28) also exists globally. Moreover, when Assumptions 1-2 hold and $P^{-1}$ remains uniformly bounded, there exist a constant $c>0$ possibly dependent on $P_{0}$, and positive constants $r<1, \gamma_{d}, \gamma_{1}, \ldots, \gamma_{N}$ such that

$$
\begin{aligned}
\left\|\tilde{x}\left(t_{k}\right)\right\| \leq & c r^{k}\|\tilde{x}(0)\|+\gamma_{d} \sup _{\tau \in\left(0, t_{k}\right)}\|\mathbf{d}(\tau)\| \\
& +\sum_{j=1}^{N} \gamma_{j} \sup _{\tau \in\left(0, t_{k}\right)}\left\|\overline{\mathbf{n}}_{j}(\tau)\right\| \quad t_{k} \geq 0
\end{aligned}
$$

where $\tilde{x}(t):=\hat{x}(t)-x(t)$ denotes the state estimation error.

Proof: See the Appendix.

As before, some condition on the observability of (1), (22) should be needed to achieve convergence of the state estimate $\hat{x}$ to the state $x$. In Theorem 4 this condition appears in the form of the requirement that $P^{-1}$ remains bounded. The following result provides a condition under which this happens.

Lemma 2: The matrix $P^{-1}$ remains uniformly bounded along trajectories of the system (1), (22), and the stateestimator (25)-(28), provided that there exist positive constants $\mathbf{N}, \epsilon$ such that the following persistence of excitation condition

$$
\left.\frac{1}{\mathbf{N}} \sum_{j=0}^{\mathbf{N}} \Phi\left(t_{i+j}, t_{i}\right)^{\prime} W\left(t_{i+j}\right) \Phi\left(t_{i+j}, t_{i}\right)\right) \geq \epsilon I>0
$$

$i=0,1, \ldots, k$, holds, where $\Phi(t, \tau)$ denotes the state transition matrix of $\dot{z}=A(u) z$.

Proof: See the Appendix.

Combining Theorem 4 and Lemma 2 we obtain the following:

Corollary 2: When Assumptions 1-2 hold, and there exist constants $\mathbf{N}, \epsilon$ such that the persistence of excitation condition (43) holds, the state estimate $\hat{x}$ converges to the state $x$ in the absence of disturbance input and measurement noise. When the disturbance and noise are bounded but nonzero, $\hat{x}$ converges to a neighborhood of the true state $x$.

\section{RIGID BODY MOTION ESTIMATION USING CCD CAMERAS}

In this section we show how one can estimate the position and orientation of a mobile robot using a CCD camera mounted on-board to observe the apparent motion of stationary points. We do this by reducing the problem to the estimation of the state of a system with perspective outputs.

Consider a coordinate frame $\{b\}$ attached to a rigid body that moves with respect to an inertial frame $\{i\}$. We denote by $\mathrm{SE}(3)$ the Cartesian product of $\mathbb{R}^{3}$ with the group $\mathrm{SO}(3)$ of $3 \times 3$ rotation matrices; and by se $(3)$ the Cartesian product of $\mathbb{R}^{3}$ with the space so(3) of $3 \times 3$ skew-symmetric matrices. Let $\left(p_{i b}, R_{i b}\right) \in \mathrm{SE}(3)$ be the configuration of the frame $\{b\}$ with respect to $\{i\}$, and $q_{1}^{i}$ and $q_{1}^{b}$ the coordinates of a point $Q_{1}$ in the frames $\{i\}$ and $\{b\}$, respectively. Then, we have that

$$
q_{1}^{i}=p_{i b}+R_{i b} q_{1}^{b}
$$


Moreover, if $q_{j}^{i}$ and $q_{j}^{b}$ denote the coordinates of another point $Q_{j}$ in the frames $\{i\}$ and $\{b\}$, respectively, we conclude that

$$
q_{j}^{b}=R_{i b}^{\prime} q_{j}^{i}-R_{i b}^{\prime} p_{i b}=R_{i b}^{\prime}\left(q_{j}^{i}-q_{1}^{i}\right)+q_{1}^{b} .
$$

We denote by $\left(v_{i b}^{b}, \Omega_{i b}^{b}\right) \in \operatorname{se}(3)$ the twist that defines the velocity of frame $\{b\}$ with respect to $\{i\}$, expressed in the frame $\{b\}$, i.e.,

$$
\Omega_{i b}^{b}=R_{i b}^{\prime} \dot{R}_{i b} \quad v_{i b}^{b}=R_{i b}^{\prime} \dot{p}_{i b} .
$$

From these two equations and (44), we obtain

$$
\dot{q}_{1}^{b}=-\Omega_{i b}^{b} q_{1}^{b}-v_{i b}^{b}+R_{i b}^{\prime} \dot{q}_{1}^{i} \quad \dot{R}_{i b}=R_{i b} \Omega_{i b}^{b} .
$$

Suppose that a camera attached to the body frame $\{b\}$ sees $N$ points $Q_{1}, Q_{2}, \ldots, Q_{N}$. Denoting by $y_{j} \in \mathbb{R}^{3}$ the homogeneous image coordinates provided by the camera of the point $Q_{j}$, the dynamics of the system can be described by the following system with $N$ perspective outputs:

$$
\begin{aligned}
\dot{q}_{1}^{b} & =-\Omega_{i b}^{b} q_{1}^{b}+R_{i b}^{\prime} \dot{q}_{1}^{i}-v_{i b}^{b} \\
\dot{R}_{i b}^{\prime} & =-\Omega_{i b}^{b} R_{i b}^{\prime} \\
\alpha_{j} y_{j} & =F\left(p_{c b}+R_{c b} q_{1}^{b}+R_{c b} R_{i b}^{\prime}\left(q_{j}^{i}-q_{1}^{i}\right)\right)
\end{aligned}
$$

$\forall j \in\{1,2, \ldots, N\}$, where $\left(p_{c b}, R_{c b}\right) \in \mathrm{SE}(3)$ denotes the configuration of the frame $\{b\}$ with respect to the camera's frame $\{c\}$, and $F$ an upper triangular matrix with the camera's intrinsic parameters, of the form $\left[\begin{array}{ccc}f_{11} & f_{12} & f_{13} \\ 0 & f_{22} & f_{23} \\ 0 & 0 & 1\end{array}\right]$, where each $f_{i j}$ denotes a scalar [32, Chapter 3]. Note that $F$ and $\left(p_{c b}, R_{c b}\right)$ can be time-varying in case the camera is allowed to zoom or pan and tilt, which is often needed to get good visual information. The normalization constraints (3) are given by $\left[\begin{array}{lll}0 & 0 & 1\end{array}\right] y_{j}=1, \forall j \in\{1,2, \ldots, N\}$.

To proceed we use the following notation: Given an $m \times n$ matrix $M$, we denote by $\operatorname{St}(M)$ the $m n$-vector obtained from stacking the columns of $M$ on top of each other, with the first column on top. Given two matrices $M_{i} \in \mathbb{R}^{m_{i} \times n_{i}}, i \in\{1,2\}$ we denote by $M_{1} \otimes M_{2} \in \mathbb{R}^{m_{1} m_{2} \times n_{1} n_{2}}$ the Kronecker product of $M_{1}$ by $M_{2}$. Using the fact that given three matrices $A, B$, $X$ with appropriate dimensions, $\operatorname{St}(A X B)=\left(B^{\prime} \otimes A\right) \operatorname{St}(X)$ [33], we can rewrite (45)-(47) as follows:

$$
\begin{aligned}
\dot{q}_{1}^{b} & =-\Omega_{i b}^{b} q_{1}^{b}+\left(\dot{q}_{1}^{i \prime} \otimes I_{3 \times 3}\right) \operatorname{St}\left(R_{i b}^{\prime}\right)-v_{i b}^{b} \\
\operatorname{St}\left(\dot{R}_{i b}^{\prime}\right) & =\left(I_{3 \times 3} \otimes-\Omega_{i b}^{b}\right) \operatorname{St}\left(R_{i b}^{\prime}\right) \\
\alpha_{j} y_{j} & =F p_{c b}+F R_{c b} q_{1}^{b}+\left(\left(q_{j}^{i}-q_{1}^{i}\right)^{\prime} \otimes F R_{c b}\right) \operatorname{St}\left(R_{i b}^{\prime}\right) .
\end{aligned}
$$

Thus, defining $x$ to be a 12-dimensional vector whose first 3 entries are the entries of $q_{1}^{b}$ and the remaining 9 entries are the columns of $R_{i b}^{\prime}$ stacked on top of each other, that is,

$$
x:=\left[\begin{array}{c}
q_{1}^{b} \\
\operatorname{St}\left(R_{i b}^{\prime}\right)
\end{array}\right] \in \mathbb{R}^{12}
$$

it follows that system (45)-(47) can be expressed in the form (1)-(2) with

$$
\begin{array}{rlrl}
A & :=\left[\begin{array}{cc}
-\Omega_{i b}^{b} & \dot{q}_{1}^{i}{ }^{\prime} \otimes I_{3 \times 3} \\
0_{9 \times 3} & I_{3 \times 3} \otimes-\Omega_{i b}^{b}
\end{array}\right] & b & :=\left[\begin{array}{c}
-v_{i b}^{b} \\
0_{9 \times 3}
\end{array}\right] \\
C_{j} & :=\left[\begin{array}{ll}
F R_{c b}\left(q_{j}^{i}-q_{1}^{i}\right)^{\prime} \otimes F R_{c b}
\end{array}\right] \quad d_{j}:=F p_{c b} \quad \forall j \in\{1, \ldots, N\} .
\end{array}
$$

and $u:=\left(v_{i b}^{b \prime}, \omega_{i b}^{b \prime}\right)^{\prime}$, where $\omega_{i b}^{b}$ is the angular velocity that parameterizes $\Omega_{i b}^{b}$. We can now use the results given in the previous sections to compute estimates $\hat{R}_{i b}^{\prime}$ and $\hat{q}_{1}^{b}$ for $R_{i b}^{\prime}$ and $q_{1}^{b}$, respectively. From $\hat{R}_{i b}^{\prime}$ and $\hat{q}_{1}^{b}, p_{i b}$ can also be estimated using

$$
\hat{p}_{i b}=q_{1}^{i}-\hat{R}_{i b} \hat{q}_{1}^{b}
$$

\section{A. Singular configurations}

Depending on the configurations of the points $Q_{1}, Q_{2}, \ldots, Q_{N}$, the state of (45)-(47) may not be observable. In fact, when all points are coplanar or collinear we can, respectively, find 9- or 6-dimensional realization for the system. However, even in this case it may still be possible to recover the position and orientation of the rigid body from the camera measurements by using the fact that $R_{i b}$ is a rotation matrix. To this effect, let $M \in \mathbb{R}^{3 \times m}$ be a matrix whose columns are a basis for the vector space generated by the $N-1$ vectors $\left\{q_{j}^{i}-q_{1}^{i}: j=2, \ldots, N\right\}$, and let $\tilde{q}_{j}$, $j \in\{2, \ldots, N\}$ be such that

$$
q_{j}^{i}-q_{1}^{i}=M \tilde{q}_{j}
$$

In this case, system (45)-(47) can be rewritten as

$$
\begin{aligned}
\dot{q}_{1}^{b} & =-\Omega_{i b}^{b} q_{1}^{b}+R_{i b}^{\prime} \dot{q}_{1}^{i}-v_{i b}^{b} \\
\dot{\bar{R}} & =-\Omega_{i b}^{b} \bar{R} \\
\alpha_{j} y_{j} & =F\left(p_{c b}+R_{c b} q_{1}^{b}+R_{c b} \bar{R} \tilde{q}_{j}\right) \quad \forall j \in\{1,2, \ldots, N\}
\end{aligned}
$$

where $\bar{R}:=R_{i b}^{\prime} M \in \mathbb{R}^{3 \times m}$. Note that when $m=\operatorname{rank} M<$ 3 , the system (45)-(47) is not observable because its inputoutput map is consistent with that of the lower-dimensional model (48)-(50).

To compute an estimate $\hat{R}_{i b}$ of $R_{i b}$ from the estimate $\hat{\bar{R}}$ of $\bar{R}$, the following two cases should be considered separately. For simplicity we assume that $M$ was chosen orthonormal, i.e., that $M^{\prime} M=I$.

1) $\operatorname{rank} M=3$, which corresponds to the existence of 4 non-coplanar points. In this case $\hat{R}_{i b}$ can be recovered directly from $\hat{\bar{R}}$ using $\hat{R}_{i b}^{\prime}=\hat{\bar{R}} M^{-1}$.

2) $\operatorname{rank} M=2$, which corresponds to all points being coplanar but not collinear. Denoting by $M^{\perp} \in \mathbb{R}^{3 \times(3-m)}$ a matrix whose columns form an orthonormal basis for the orthogonal complement of the image of $M$ (i.e., a full rank matrix such that $M^{\perp^{\prime}} M^{\perp}=I$ and $M^{\prime} M^{\perp}=0$ ), the general solution to $M^{\prime} \hat{R}_{i b}=\hat{\bar{R}}^{\prime}$, is of the form

$$
\hat{R}_{i b}=M \hat{\bar{R}}^{\prime}+M^{\perp} \mu^{\prime}
$$

for some vector $\mu \in \mathbb{R}^{3 \times(3-m)}$. This vector needs to be determined from the fact that $\hat{R}_{i b}^{\prime}$ is orthonormal. Since

$$
\hat{R}_{i b}^{\prime} \hat{R}_{i b}=\left(\hat{\bar{R}} M^{\prime}+\mu M^{\perp^{\prime}}\right)\left(M \hat{\bar{R}}^{\prime}+M^{\perp} \mu^{\prime}\right)=\hat{\bar{R}} \hat{\bar{R}}^{\prime}+\mu \mu^{\prime}
$$

we conclude that $\mu$ needs to be chosen to make $\hat{\bar{R}} \hat{\bar{R}}^{\prime}+\mu \mu^{\prime}$ as close to the identity as possible.

A straightforward way to compute the vector $\mu$ is to minimize the Frobenius norm $\|\cdot\|_{F}$ of

$$
\begin{array}{r}
\left\|\hat{\bar{R}} \hat{\bar{R}}^{\prime}+\mu \mu^{\prime}-I\right\|_{F}^{2}:=\operatorname{Tr}\left(\hat{\bar{R}} \hat{\bar{R}}^{\prime}+\mu \mu^{\prime}-I\right)^{\prime}\left(\hat{\bar{R}} \hat{\bar{R}}^{\prime}+\mu \mu^{\prime}-I\right) \\
=\|\mu\|^{4}+2 \mu^{\prime}\left(\hat{\bar{R}} \hat{\bar{R}}^{\prime}-I\right) \mu+\operatorname{Tr}\left(\hat{\bar{R}} \hat{\bar{R}}^{\prime}-I\right)^{2} .
\end{array}
$$


Denoting by $\lambda$ the most negative eigenvalue of $\hat{\bar{R}} \hat{\bar{R}}^{\prime}-I$ (which must be negative since any vector in the kernel of $\hat{\bar{R}}^{\prime}$ is an eigenvector corresponding to the eigenvalue -1 ) and by $v \in \mathbb{R}^{3}$ the corresponding unit-norm eigenvector, the previous expression has a minimum at $\mu^{\prime}=\alpha v$, which is equal to

$$
\alpha^{4}+2 \alpha^{2} \lambda+\operatorname{Tr}\left(\hat{\bar{R}} \hat{\bar{R}}^{\prime}-I\right)^{2}
$$

where $\alpha$ is a scalar. The minimum is then obtained for $\alpha^{2}=$ $-\lambda$ and therefore $\mu= \pm \sqrt{-\lambda} v, \hat{R}_{i b}=M \hat{\bar{R}}^{\prime} \pm \sqrt{-\lambda} M^{\perp} v^{\prime}$. The sign for the square root can be determined from the constraint that the determinant of $\hat{R}_{i b}$ be positive. Note that $\mu$ is unique as long as $\hat{\bar{R}} \hat{\bar{R}}^{\prime}-I$ does not have more than one eigenvector associated with the most negative eigenvalue.

When all points are collinear there is a fundamental loss of observability that cannot be overcome without extra information. Indeed, when there is a direction $v \in \mathbb{R}^{3}$ such that all line segments between all points are aligned with $v$, i.e.,

$$
q_{j_{1}}^{i}-q_{j_{2}}^{i}=\beta_{j_{1} j_{2}} v \quad \forall j_{1}, j_{2} \in\{1,2, \ldots, N\}, \beta_{j_{1} j_{2}} \in \mathbb{R}
$$

and the velocity of the points (if any) is also aligned with $v$, i.e., $\dot{q}_{j}^{i}=\gamma_{j} v, \forall j \in\{1,2, \ldots, N\}, \gamma_{j} \in \mathbb{R}$, the matrix $R_{v} \in \mathrm{SO}(3)$ that corresponds to a fixed rotation around $v$ has the property that

$$
R_{v} \dot{q}_{1}^{i}=\dot{q}_{1}^{i} \quad R_{v}\left(q_{j}^{i}-q_{1}^{i}\right)=q_{j}^{i}-q_{1}^{i} \quad \forall j \in\{1,2, \ldots, N\}
$$

In this case, the system (48)-(50) with $\bar{R}_{i b}^{\prime}:=R_{i b}^{\prime} R_{v}$ has exactly the same input-output map as (45)-(47). This means that $R_{i b}$ can only be recovered from the perspective outputs up to a rotation around $v$.

Remark 5: In [34], we incorporated quadratic state constraints in the minimum-energy formulation. When this is done, there is no need to consider lower dimensional realizations for (45)-(47) when the points are coplanar but not collinear.

\section{B. Unknown inertial coordinates}

Suppose now that the inertial coordinates of the points $Q_{1}, Q_{2}, \ldots, Q_{N}$ are not known. In this case, one can still estimate $x$ by using three of the points to define an inertial coordinate system. To this effect, let

$$
S:=R_{i b}^{\prime}\left[\begin{array}{llll}
q_{2}^{i}-q_{1}^{i} & q_{3}^{i}-q_{1}^{i} \quad \cdots \quad q_{N}^{i}-q_{1}^{i}
\end{array}\right] .
$$

We can rewrite (45)-(47) as

$$
\begin{aligned}
\dot{q}_{1}^{b} & =-\Omega_{i b}^{b} q_{1}^{b}+R_{i b}^{\prime} \dot{q}_{1}^{i}-v_{i b}^{b} \\
\dot{S} & =-\Omega_{i b}^{b} S \\
\alpha_{j} y_{j} & =F\left(p_{c b}+R_{c b} q_{1}^{b}+R_{c b} S \tilde{q}_{j}\right) \quad \forall j \in\{1,2, \ldots, N\}
\end{aligned}
$$

where $e_{j}$ denotes the $j$ th column of the $(N-1) \times(N-1)$ identity matrix.

To recover an estimate $\hat{R}_{i b}$ of $R_{i b}$ from the estimate $\hat{S}$ of $S$, we can use the QR decomposition to obtain a rotation matrix $\hat{R}_{i b}$ and an upper triangular matrix $\hat{U}$ such that

$$
\hat{S}=\hat{R}_{i b}^{\prime} \hat{U}
$$

and then, defining $q_{1}^{i}:=0$ and $q_{j}^{i}, j \in\{2,3, \ldots, N\}$ equal to the $(j-1)$ th column of $\hat{U}$. This corresponds to the following

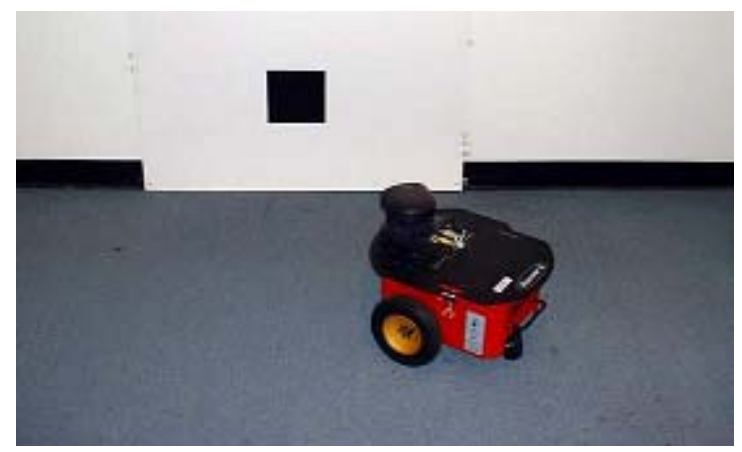

Fig. 1. Experimental setup: Pionner 2-DXE mobile robot with CCD camera mounted on top and visual landmark.

convention to construct the inertial coordinate system: the origin of $\{i\}$ is the point $Q_{1}$; its first axis is defined by the direction from $Q_{1}$ to $Q_{2}$; its second axis is orthogonal to the first one and lies on the plane defines by $Q_{1}, Q_{2}$, and $Q_{3}$; and its third axis is defined by the cross product of the first two.

\section{Experimental results}

The theoretical results presented in the previous sections were experimentally validated by applying them to estimate the position and orientation of a mobile robot using measurements from an on-board CCD camera. This section describes the experimental setup and presents the results obtained for three types of experiments: robot stopped, following a circular path, and parking.

The experiments were carried out on a Pionner 2-DXE mobile robot from ActivMedia [35]. The vehicle, shown in Fig. 1 , has two rear wheels which are powered by two independent high torque, reversible-DC motors, and one passive rear caster to balance the robot. The vehicle is equipped with a Sony EVI D30 pan-tilt-zoom (PTZ) color video camera mounted on the top of the robot with its optical axis oriented towards the forward direction (when pan and tilt angles are zero). To simplify the image processing, in these experiments we used the corners of a black square as visual landmarks (see Fig. 1). The location of these points were obtained by detecting the edges of the square and then computing their intersections. A pan controller was also implemented to keep as much as possible the visual landmark in the center of the image.

1) Robot stopped experiment: To validate the minimumenergy state estimators described in Sections II-III, several open-loop tests were carried out. A simple test consists of running an estimator with the vehicle stopped and commanding the pan angle of the camera.

Fig. 2 shows the experimental results of this test using the estimator proposed in Section II. The robot is at position $(x, y)=(-0.8 \mathrm{~m}, 0 \mathrm{~m})$ with $\theta=0 \mathrm{rad}$. The pan angle is set to zero. At $t=20 \mathrm{~s}$ a ramp-like signal is applied in pan until $t=30 \mathrm{~s}$. As shown in the figure, the estimator converges to the true values and is not affected by the camera's pan motion.

2) Following a circular path experiment: To illustrate the estimator proposed in Section III, we present here results for a test in which the vehicle follows a circular path. Since the pan angle is limited to $\left[-\frac{\pi}{2}, \frac{\pi}{2}\right]$, the visual landmarks periodically 


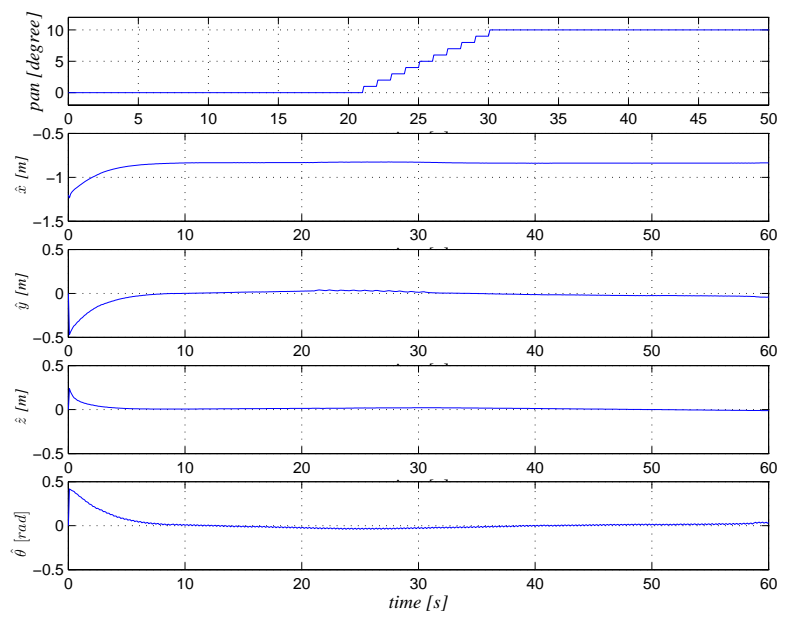

Fig. 2. Robot stopped experiment: Time evolution of pan angle and estimator outputs $\hat{p}_{i b}:=(\hat{x}, \hat{y}, \hat{z})^{\prime}$ and $\hat{\theta}$.

leave the camera's field of view. While this happens, the estimator does not receive any visual measurements.

Fig. 3(a)-3(c) show the experimental results. We can see that the output of the estimator converges to the values correspondent to a circular trajectory. Another interesting observation is the behavior of the pan controller that is always trying to compensate the motion of the robot in order to keep the features in the image $3(\mathrm{~b})$.

Our experimental setup does not provide us with ground truth information to contrast with the estimates obtained by the observer. To provide some insight into a typical evolution of the estimation error we show in Fig. 4 results obtained in a Matlab simulation with noise levels similar to those in the experimental setup. It can be seen that the estimated pose tends to a small neighborhood of the true value. Observe also, as expected, that the estimation errors only reduce significantly when the visual landmarks are in the camera's field of view.

3) Parking experiment: The nonholonomic kinematics model of the Pionner 2-DXE is given by

$$
\dot{x}=v \cos (\theta), \quad \dot{y}=v \sin (\theta), \quad \dot{\theta}=\omega,
$$

where $v$ and $\omega$ denote the linear and angular velocity, respectively. In this section we present experimental results obtained using the minimum-energy state estimator described in Section II combined with a pan controller and the point stabilization controller presented in [36]. The stabilization control law used is a piecewise time-invariant continuous feedback law and it is based on a non-smooth state transformation inspired by the polar description of the kinematics of the robot. This particular controller was chosen because its control strategy is intuitive, simple to implement, offers a good performance, and the resulting paths are fairly natural, i.e., similar to what a human operator would attempt. However, as reported in [37], this controller is inherently sensitive to sensor noise and small perturbations around the origin.

Fig. 5(a)-5(c) show the experimental results for the case where the vehicle starts stationary at $(x, y)=(-0.8 \mathrm{~m}, 0.4 \mathrm{~m})$ and with heading $\theta=0$. The objective was to park the vehicle at position $(x, y)=(-0.5 \mathrm{~m}, 0 \mathrm{~m})$ with heading $\theta=0$.
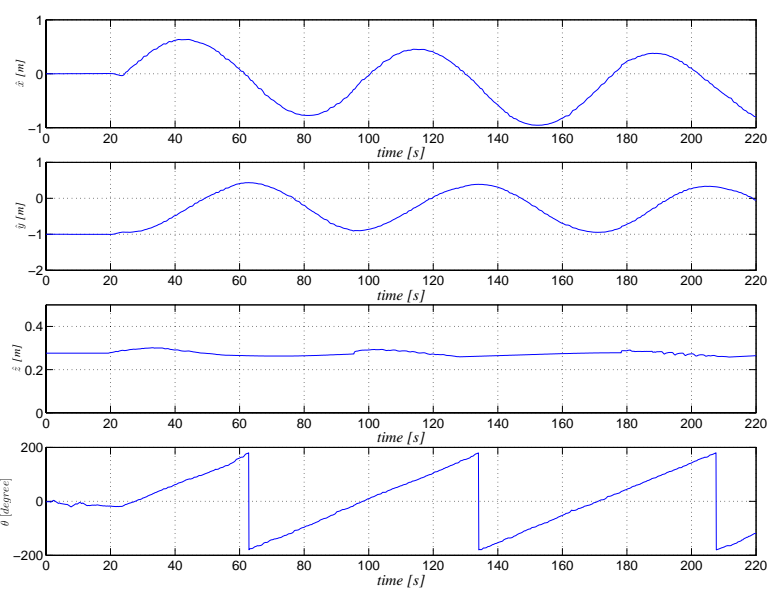

(a)
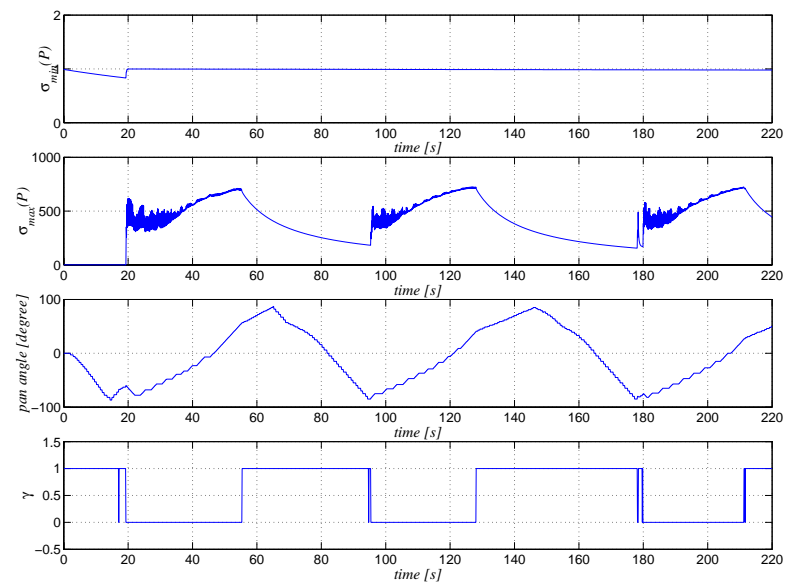

(b)
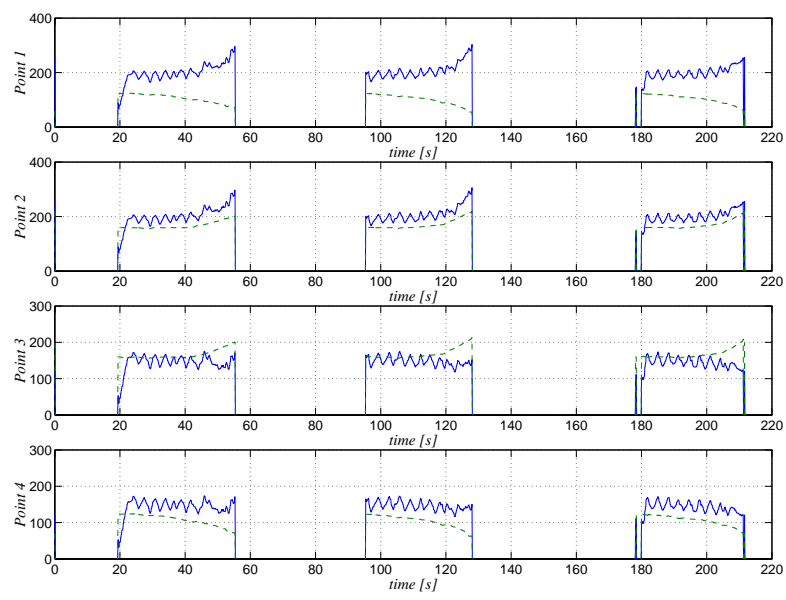

(c)

Fig. 3. Following a circular path experiment: Time evolution of 3(a) the estimated position $(\hat{x}, \hat{y}, \hat{z})$, and orientation $\hat{\theta} ; 3(\mathrm{~b})$ the minimum and maximum singular values of $P$, respectively; the pan angle; and the variable $\gamma$ which indicates when the estimator is receiving $(\gamma=0)$ or not $(\gamma=1)$ measurements; and 3(c) the position in the image of the corners of the visual landmark $(\mathrm{x}-, \mathrm{y}--)$. When the points are out of the camera's field of view, the points' coordinates are not shown.

Fig. 5(a) displays the resulting vehicle trajectory computed based on dead-reckoning data measurements. Note that deadreckoning only gives the pose of the mobile robot with respect 

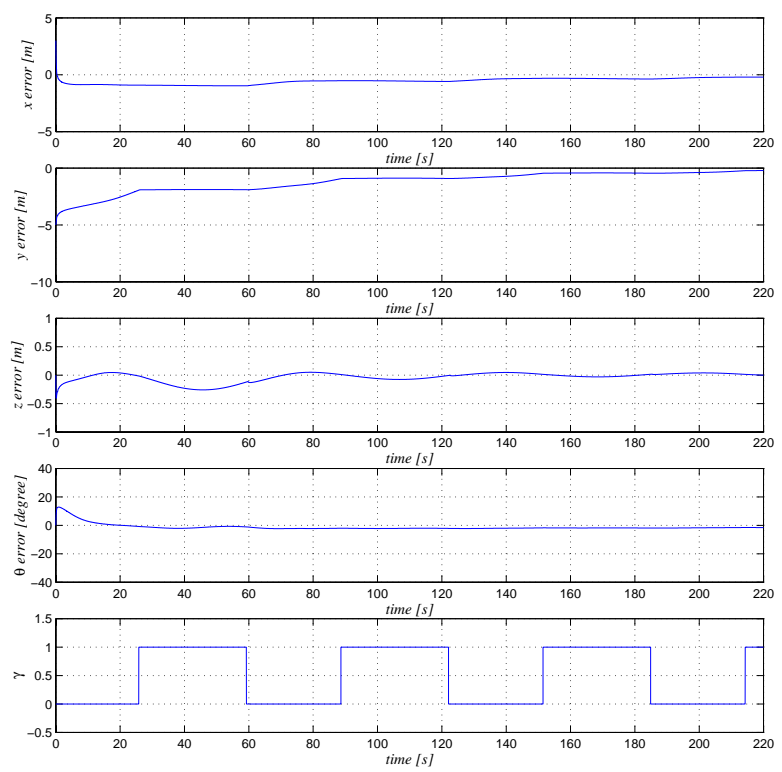

Fig. 4. Simulation results for the circular path. Time evolution of the estimation errors (position and orientation), and the signal $\gamma$, which indicates when the estimator is receiving $(\gamma=0)$ or not $(\gamma=1)$ measurements.

to the starting initial condition and is therefore not useful to park at a point specified in a referential frame defined by visual landmarks. Fig. 5(b) shows the time evolution of estimator outputs $\hat{p}_{i b}$ and $\hat{\theta}$, and Fig. 5(c) the time evolution of control signals $u_{1}, u_{2}$, and $u_{\text {pan }}$. In this experiment we imposed that the point stabilization control algorithm only starts to operate at time $t=5 \mathrm{~s}$ and the pan controller at time $t=1 \mathrm{~s}$. As expected, the vehicle converges to a very small neighborhood of the desired pose. From Fig. 5(c) it can be seen that the pan controller is indeed able to compensate the motion of the robot in order to keep the features in the center of the image.

\section{CONCLUSions}

We considered the problem of estimating the state of a system with perspective outputs either available continuously, or only at discrete time instants with some delay and perhaps incomplete. We designed estimators that are globally convergent under appropriate observability assumptions and can therefore, be used to design output-feedback controllers. We applied these results to estimate the position and orientation of a mobile robot using measurements from an attached CCD camera. The estimator proposed requires the robot's linear and angular velocities. Adaptive estimation techniques can probably be used to estimate these parameters when they are not available. This, the investigation of observability assumptions less restrictive than (20) or (43), and the design of statefeedback controllers that satisfy the required observability assumptions are the subject of our future research. Another topic for future research is to incorporate algebraic constraints on the state in the estimation algorithm. Preliminary results regarding this issue can be found in [34], where we incorporated quadratic state constraints in the minimum-energy formulation.

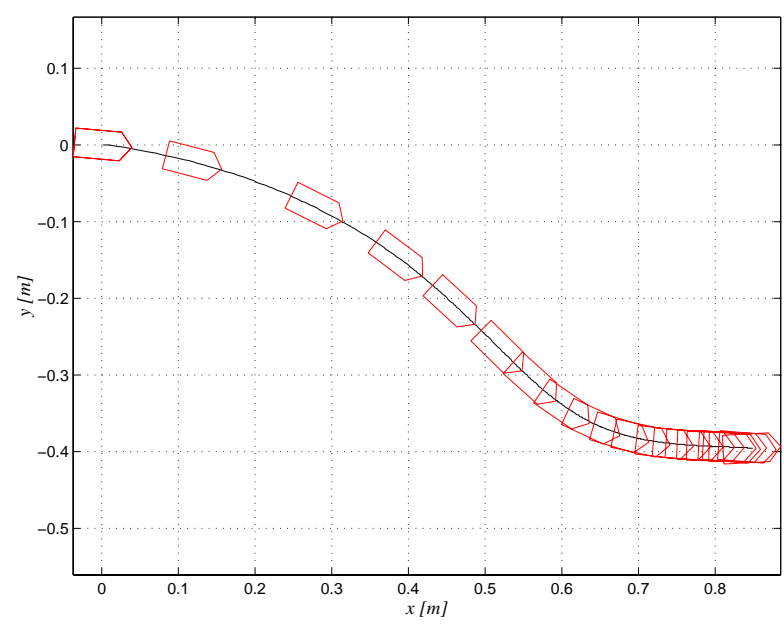

(a)
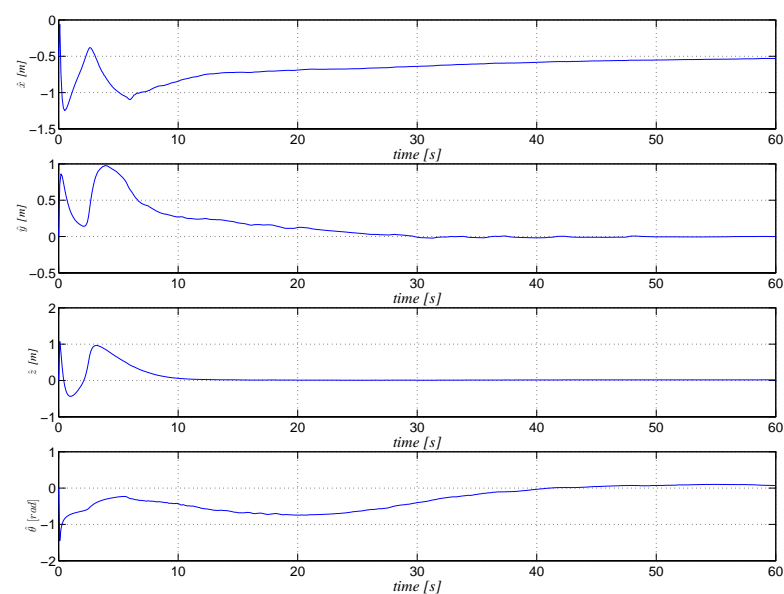

(b)
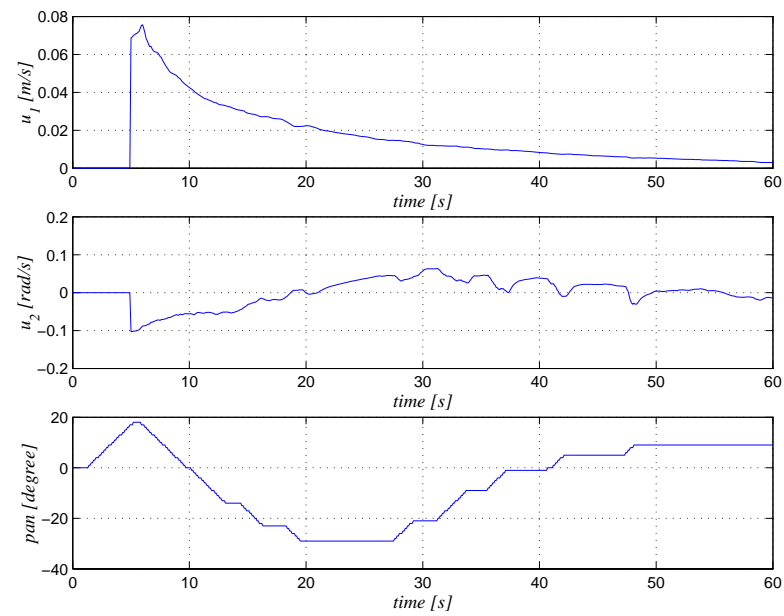

(c)

Fig. 5. Parking experiment: 5(a) Resulting trajectory of the mobile robot in the $x y$-plane using dead-reckoning data measurements; Time evolution of 5(b) estimator outputs $\hat{p}_{i b}$ and $\hat{\theta}$; and 5(c) control signals $u_{1}(t), u_{2}(t)$, and $u_{\text {pan }}(t)$.

\section{APPENDIX}

Claim 1: The matrix $P(t)$ governed by (17) is positive definite for all $t \geq 0$. 
Proof: Observe that (17) can also be written as $\dot{P}=-P\left(A+G G^{\prime} P\right)-\left(A+G G^{\prime} P\right)^{\prime} P+P G G^{\prime} P+W$

and, therefore,

$$
\begin{aligned}
P(t)= & \Psi(0, t)^{\prime} P_{0} \Psi(0, t) \\
& +\int_{0}^{t} \Psi(\tau, t)^{\prime}\left(P G G^{\prime} P+W\right) \Psi(\tau, t) d \tau \quad t \geq 0
\end{aligned}
$$

where $\Psi(t, \tau)$ denotes the state transition matrix of $\dot{z}=(A+$ $\left.G G^{\prime} P\right) z$. This can be verified by taking derivatives of the candidate expression (54) for $P$ :

$$
\begin{aligned}
\dot{P}= & -\left(A+G G^{\prime} P\right)^{\prime} \Psi(0, t)^{\prime} P_{0} \Psi(0, t) \\
& -\Psi(0, t)^{\prime} P_{0} \Psi(\tau, t)\left(A+G G^{\prime} P\right)+P G G^{\prime} P+W \\
& -\left(A+G G^{\prime} P\right)^{\prime} \int_{0}^{t} \Psi(\tau, t)^{\prime}\left(P G G^{\prime} P+W\right) \Psi(\tau, t) d \tau \\
& -\int_{0}^{t} \Psi(\tau, t)^{\prime}\left(P G G^{\prime} P+W\right) \Psi(\tau, t) d \tau\left(A+G G^{\prime} P\right) \\
= & -\left(A+G G^{\prime} P\right)^{\prime} P-P\left(A+G G^{\prime} P\right)+P G G^{\prime} P+W .
\end{aligned}
$$

Here, we used the fact that, for every fixed $\tau$,

$$
\begin{aligned}
\frac{\mathrm{d}}{\mathrm{d} t} \Psi(\tau, t) & =-\Psi(t, \tau)^{-1}\left(\frac{\mathrm{d}}{\mathrm{d} t} \Psi(t, \tau)\right) \Psi(t, \tau)^{-1} \\
& =-\Psi(t, \tau)^{-1}\left(A+G G^{\prime} P\right) \Psi(t, \tau) \Psi(t, \tau)^{-1} \\
& =-\Psi(\tau, t)\left(A+G G^{\prime} P\right) .
\end{aligned}
$$

Now, since $\Psi(t, 0) P_{0} \Psi(t, 0)^{\prime}>0$ and $P G G^{\prime} P+W \geq 0$, from (54) we conclude that $P(t)$ remains positive definite for all $t \geq 0$.

Proof: [Theorem 2] From (1) and (9) we conclude that

$$
\dot{\tilde{x}}=(A-Q W) \tilde{x}-G \mathbf{d}+Q \sum_{j=1}^{N} C_{j}^{\prime} Y_{j}^{\prime} Y_{j} \mathbf{n}_{j}
$$

where each $Y_{j}$ is a matrix for which $I-\frac{y_{j} y_{j}^{\prime}}{\left\|y_{j}\right\|^{2}}=Y_{j}^{\prime} Y_{j}$. Such matrices always exist because $I-\frac{y_{j} y_{j}^{\prime}}{\left\|y_{j}\right\|^{2}} \geq 0$. Defining $V(\tilde{x}):=$ $\tilde{x}^{\prime} P \tilde{x}, P:=Q^{-1}$, computing its time-derivative, and using (17), we get

$$
\dot{V}=-\tilde{x}^{\prime}\left(P G G^{\prime} P+W\right) \tilde{x}-2 \tilde{x}^{\prime} P G \mathbf{d}+2 \tilde{x}^{\prime} \sum_{j=1}^{N} C_{j}^{\prime} Y_{j}^{\prime} Y_{j} \mathbf{n}_{j}
$$

By completing the squares, we further conclude that

$$
\begin{aligned}
\dot{V} & \leq-\frac{1}{2} \tilde{x}^{\prime}\left(P G G^{\prime} P+W\right) \tilde{x}+2\|\mathbf{d}\|^{2}+2 \sum_{j=1}^{N} \mathbf{n}_{j}^{\prime} Y_{j}^{\prime} Y_{j} \mathbf{n}_{j} \\
& \leq-\frac{1}{2} \tilde{x}^{\prime}\left(P G G^{\prime} P+W\right) \tilde{x}+2\|\mathbf{d}\|^{2}+2 \sum_{j=1}^{N}\left\|\mathbf{n}_{j}\right\|^{2}
\end{aligned}
$$

where we used the fact that the largest eigenvalue of $Y_{j}^{\prime} Y_{j}$ is always smaller than 1 . Since for every finite time, $P$ is positive definite, $V$ must be finite on any finite interval and, therefore, so must be $\tilde{x}$ and $\hat{x}$. Global existence of solution follows. In case $Q$ in uniformly bounded, $P$ is uniformly positive definite and so is $P G G^{\prime} P$ (by Assumption 1). In this case,

$$
\dot{V} \leq-\frac{1}{2} \delta \mu V+2\|\mathbf{d}\|^{2}+2 \sum_{j=1}^{N}\left\|\mathbf{n}_{j}\right\|^{2}
$$

where $\mu>0$ is some constant that satisfies $P(t) \geq \mu I, \forall t \geq$ 0 . It is now straightforward to conclude that the ISS-like bound (19) holds.

Proof: [Lemma 1] To prove this lemma we show that

$$
P(t) \geq \mu I>0 \quad \forall t \geq 0
$$

for some positive constant $\mu$. To this effect, we pick an arbitrary vector $x \in \mathbb{R}^{n}$ and compute $x^{\prime} P(t) x$ using (54):

$$
\begin{aligned}
x^{\prime} P(t) x= & z(0)^{\prime} P_{0} z(0) \\
& +\int_{0}^{t} z(\tau)^{\prime}\left(P(\tau) G G^{\prime} P(\tau)+W(\tau)\right) z(\tau) d \tau
\end{aligned}
$$

where $z(\tau):=\Psi(\tau, t) x, \tau \leq t$ is the solution to

$$
\dot{z}(\tau)=\left(A+G G^{\prime} P\right) z(\tau) \quad z(t)=x \quad 0 \leq \tau \leq t .
$$

Since $P_{0}>0$, then for $t \geq T$, we conclude that

$$
x^{\prime} P(t) x \geq \alpha(t)^{2}+\beta(t)^{2} \quad \forall t \geq T
$$

where $\quad \alpha(t) \quad:=\left\|W(\tau)^{\frac{1}{2}} z(\tau)\right\|_{(t-T, t)}$, and $\beta(t) \quad:=$ $\left\|G^{\prime} P(\tau) z(\tau)\right\|_{(t-T, t)}$. Here, given a positive semidefinite matrix $M$ we denote by $M^{\frac{1}{2}}$ any matrix such that $\left(M^{\frac{1}{2}}\right)^{\prime} M^{\frac{1}{2}}=$ $M$ and given a signal $x$ we denote by $\|x\|_{(a, b)}$ the $\mathcal{L}_{2^{-}}$ norm of $x$ truncated to the interval $(a, b)$, i.e., $\|x\|_{(a, b)}:=$ $\left(\int_{a}^{b}\|x(\tau)\|^{2} d \tau\right)^{\frac{1}{2}}$. We now proceed to compute a lower-bound for $x^{\prime} P(t) x$ by computing a lower-bound for the right-handside of (58). Fix some $t \geq T$. Rewriting (57) as $\dot{z}=$ $A z+G G^{\prime} P z$ and using the variation of constants formula, it follows that

$$
z(\tau)=\Phi(\tau, t) x+\int_{t}^{\tau} \Phi(\tau, s) G G^{\prime} P(s) z(s) d s \quad 0 \leq \tau \leq t .
$$

Therefore,

$$
\begin{aligned}
& \left\|W(\tau)^{\frac{1}{2}} \Phi(\tau, t) x\right\|_{(t-T, t)} \\
& \quad \leq \alpha+\left\|\int_{t}^{\tau} W(\tau)^{\frac{1}{2}} \Phi(\tau, s) G G^{\prime} P(s) z(s) d s\right\|_{(t-T, t)}
\end{aligned}
$$

Moreover, using the Schwartz inequality [38], we conclude that

$$
\begin{aligned}
& \left\|\int_{t}^{\tau} W(\tau)^{\frac{1}{2}} \Phi(\tau, s) G G^{\prime} P(s) z(s) d s\right\|_{(t-T, t)}^{2} \\
& =\int_{t-T}^{t}\left\|\int_{t}^{\tau} W(\tau)^{\frac{1}{2}} \Phi(\tau, s) G G^{\prime} P(s) z(s) d s\right\|^{2} d \tau \\
& \leq \int_{t-T}^{t}\left(\int_{\tau}^{t}\left\|W(\tau)^{\frac{1}{2}} \Phi(\tau, s) G\right\|^{2} d s \int_{\tau}^{t}\left\|G^{\prime} P(s) z(s)\right\|^{2} d s\right) d \tau
\end{aligned}
$$

and because $\left\|W(\tau)^{\frac{1}{2}} \Phi(\tau, s) G\right\|$ is uniformly bounded for $s \in$ $[\tau, t]$, we further conclude that

$$
\begin{aligned}
& \left\|\int_{t}^{\tau} W(\tau)^{\frac{1}{2}} \Phi(\tau, s) G G^{\prime} P(s) z(s) d s\right\|_{(t-T, t)}^{2} \\
& \quad \leq c \int_{t-T}^{t} \int_{\tau}^{t}\left\|G^{\prime} P(s) z(s)\right\|^{2} d s d \tau \leq c T \beta(t)^{2}
\end{aligned}
$$

for an appropriately defined constant $c$. From (59) and (60), it then follows that

$$
\left\|W(\tau)^{\frac{1}{2}} \Phi(\tau, t) x\right\|_{(t-T, t)} \leq \alpha(t)+\sqrt{c T} \beta(t) \quad \forall t \geq T .
$$


From this, (58), and assuming without loss of generality that $c T \geq 1$, it is straightforward to conclude that

$$
\begin{aligned}
x^{\prime} P(t) x \geq & \alpha^{2}+\beta^{2} \geq \frac{(\alpha+\sqrt{c T} \beta)^{2}}{2 c T} \\
\geq & \frac{1}{2 c T} \int_{t-T}^{t} x^{\prime} \Phi(\tau, t)^{\prime} W(\tau) \Phi(\tau, t) x d \tau \\
= & \frac{1}{2 c T} x^{\prime} \Phi(t-T, t)^{\prime}\left(\int_{0}^{T} x^{\prime} \Phi(t-T+s, t-T)^{\prime} \times\right. \\
& W(t-T+s) \Phi(t-T+s, t-T) d s) \Phi(t-T, t) x
\end{aligned}
$$

for $t \geq T$. From this and (20), we obtain

$$
x^{\prime} P(t) x \geq \frac{\epsilon}{2 c}\|\Phi(t-T, t) x\|^{2} \geq \frac{\epsilon}{2 c\|\Phi(t, t-T)\|^{2}}\|x\|^{2}
$$

$\forall t \geq T$. This proves that (56) holds with $\mu$ equal to the smallest of $\frac{\epsilon}{2 c\|\Phi(t, t-T)\|^{2}}$ and the smallest eigenvalue of $P(t)$ on the closed interval $[0, T]$. The latter is strictly positive since $P(t)$ is positive definite for any finite time $t$.

Proof: [Theorem 4] From (1) and (26), we conclude that for all $t_{i} \leq t<t_{i+1}$, the state estimation error evolves according to

$$
\dot{\tilde{x}}=A(u) \tilde{x}-G(u) \mathbf{d} .
$$

Defining $V:=\tilde{x} P \tilde{x}$, and computing its time derivative, it follows that

$$
\begin{aligned}
\dot{V} & =\tilde{x}^{\prime}\left(\dot{P}+P A+A^{\prime} P\right) \tilde{x}-2 \tilde{x} P G \mathbf{d} \\
& =-\tilde{x}^{\prime}\left(P G G^{\prime} P\right) \tilde{x}-2 \tilde{x}^{\prime} P G \mathbf{d} \quad t_{i} \leq t<t_{i+1}
\end{aligned}
$$

By completing the squares and using Assumption 1, we obtain

$$
\begin{aligned}
& \dot{V}=-\frac{1}{2} \tilde{x}^{\prime}\left(P G G^{\prime} P\right) \tilde{x}-\frac{1}{2}\left\|G^{\prime} P \tilde{x}+2 \mathbf{d}\right\|^{2}+2\|\mathbf{d}\|^{2} \\
& \leq-\gamma V+2\|\mathbf{d}\|^{2} \quad t_{i} \leq t<t_{i+1}
\end{aligned}
$$

where $\gamma:=\frac{1}{2} \delta \inf _{\tau \in\left[t_{i}, t_{i+1}\right)} \lambda_{\text {min }}(P(\tau))$ and $\lambda_{\min }(P)$ denotes the smallest eigenvalue of $P$. Using the assumption that $P^{-1}$ is uniformly bounded we conclude that $\gamma>0$ and

$$
V(t) \leq V\left(t_{i}\right) e^{-\gamma\left(t-t_{i}\right)}+\frac{2}{\gamma} \sup _{\tau \in\left[t_{i}, t\right)}\|\mathbf{d}(\tau)\|^{2} .
$$

Consider now $t=t_{i+1}$. From (27)-(28), the estimation error $\tilde{x}$ at time $t=t_{i+1}$ can be written as

$$
\tilde{x}\left(t_{i+1}\right)=\left[I-P\left(t_{i+1}\right)^{-1} W\left(t_{i+1}\right)\right] \tilde{x}\left(t_{i+1}^{-}\right)+P\left(t_{i+1}\right)^{-1} \eta
$$

where $\eta:=\sum_{j \in \mathcal{I}_{i+1}} \bar{C}_{j}^{\prime} Y_{j}^{\prime} Y_{j} \overline{\mathbf{n}}_{j}$, and each $Y_{j}$ is a matrix for which $I-\frac{\mathbf{y}_{j} \mathbf{y}_{j}^{\prime}}{\left\|\mathbf{y}_{j}\right\|^{2}}=Y_{j}^{\prime} Y_{j}$. Such matrices always exist because $I-\frac{\mathbf{y}_{j} \mathbf{y}_{j}^{\prime}}{\left\|\mathbf{y}_{j}\right\|^{2}} \geq 0$. Thus,

$$
\begin{aligned}
V\left(t_{i+1}\right)= & \tilde{x}\left(t_{i+1}^{-}\right)^{\prime}\left[P\left(t_{i+1}\right)-2 W\left(t_{i+1}\right)\right. \\
& \left.+W\left(t_{i+1}\right) P\left(t_{i+1}\right)^{-1} W\left(t_{i+1}\right)\right] \tilde{x}\left(t_{i+1}^{-}\right) \\
& +\eta^{\prime} P\left(t_{i+1}\right)^{-1} \eta \\
& +2 \tilde{x}\left(t_{i+1}^{-}\right)^{\prime}\left[I-W\left(t_{i+1}\right) P\left(t_{i+1}\right)^{-1}\right] \eta .
\end{aligned}
$$

Simplifying the notation by dropping the time dependence, using (27), and resorting to the matrix inversion lemma ${ }^{3}$, the first terms in brackets on the right-hand-side of (63) can be written as

$$
\begin{aligned}
P\left(t_{i+1}\right) & -2 W+W P\left(t_{i+1}\right)^{-1} W \\
& =P-W+W[P+W]^{-1} W \\
& =P-W^{\frac{1}{2}}\left[I-W^{\frac{1}{2}}\left[P+W^{\frac{1}{2}} I W^{\frac{1}{2}}\right]^{-1} W^{\frac{1}{2}}\right] W^{\frac{1}{2}} \\
& =P-W^{\frac{1}{2}} F W^{\frac{1}{2}}
\end{aligned}
$$

where $F:=\left[I+W^{\frac{1}{2}} P^{-1} W^{\frac{1}{2}}\right]^{-1}$ and $P=P\left(t_{i+1}^{-}\right)$. In this setting, given a positive semidefinite matrix $M$, we denote by $M^{\frac{1}{2}}$ any matrix such that $\left(M^{\frac{1}{2}}\right)^{\prime} M^{\frac{1}{2}}=M$. The others terms in (63) can be written as

$$
\begin{aligned}
P\left(t_{i+1}\right)^{-1} & =(P+W)^{-1} \\
& =P^{-1}-P^{-1} W^{\frac{1}{2}} F W^{\frac{1}{2}} P^{-1} \\
I-W\left(t_{i+1}\right) P\left(t_{i+1}\right)^{-1} & =I-W^{\frac{1}{2}} I W^{\frac{1}{2}}\left[P+W^{\frac{1}{2}} I W^{\frac{1}{2}}\right]^{-1} \\
& =I-W^{\frac{1}{2}} F W^{\frac{1}{2}} P^{-1} .
\end{aligned}
$$

Thus,

$$
\begin{aligned}
V\left(t_{i+1}\right)= & \tilde{x}^{\prime} P \tilde{x}-\tilde{x}^{\prime} W^{\frac{1}{2}} F W^{\frac{1}{2}} \tilde{x} \\
& +\eta^{\prime}\left(P^{-1}-P^{-1} W^{\frac{1}{2}} F W^{\frac{1}{2}} P^{-1}\right) \eta \\
& +2 \tilde{x}^{\prime}\left(I-W^{\frac{1}{2}} F W^{\frac{1}{2}} P^{-1}\right) \eta .
\end{aligned}
$$

By completing the squares, we further conclude that

$$
V\left(t_{i+1}\right) \leq(1+\epsilon) V\left(t_{i+1}^{-}\right)+\left(1+\frac{1}{\epsilon}\right) \eta^{\prime} P^{-1} \eta
$$

where $\epsilon$ is an arbitrary small positive constant. Therefore, resorting to (61), $V\left(t_{i+1}\right)$ satisfies

$$
V\left(t_{i+1}\right) \leq(1+\epsilon) V\left(t_{i}\right) e^{-\gamma\left(t_{i+1}-t_{i}\right)}+\frac{1}{\epsilon} a_{i+1}+b_{i+1}
$$

where $a_{i+1}:=\lambda_{\max }\left(P^{-1}\right)\|\eta\|^{2}$, and $b_{i+1}:=(1+$ e) $\frac{2}{\gamma} \sup _{\tau \in\left[t_{i}, t_{i+1}\right)}\|\mathbf{d}(\tau)\|^{2}+a_{i+1}$. Furthermore, solving this inequality recursively, we get

$$
\begin{aligned}
V\left(t_{k}\right) \leq & (1+\epsilon)^{k} e^{-\gamma\left(t_{k}-t_{0}\right)} V\left(t_{0}\right) \\
& +\sum_{j=0}^{k-1}(1+\epsilon)^{j} e^{-\gamma\left(t_{k}-t_{k-j}\right)}\left(\frac{1}{\epsilon} a_{k-j}+b_{k-j}\right) .
\end{aligned}
$$

Applying Assumption 2, we first notice that

$$
t_{k}-t_{k-j} \geq\left[j-N_{0}\right] \tau_{D}, \quad j=0,1, \ldots, k-1 .
$$

Consequently,

$$
\begin{aligned}
V\left(t_{k}\right) \leq & {\left[(1+\epsilon) e^{-\gamma \tau_{D}}\right]^{k} e^{\gamma N_{0} \tau_{D}} V\left(t_{0}\right) } \\
& +\sum_{j=0}^{k-1}\left[(1+\epsilon) e^{-\gamma \tau_{D}}\right]^{j}\left(\frac{1}{\epsilon} a_{k-j}+b_{k-j}\right) e^{\gamma N_{0} \tau_{D}}
\end{aligned}
$$

${ }^{3}$ Let $A, C$, and $A^{-1}+B^{\prime} C^{-1} B$ be non-singular matrices, then $\left(A^{-1}+\right.$ $\left.B^{\prime} C^{-1} B\right)^{-1}=A-A B^{\prime}\left(B A B^{\prime}+C\right)^{-1} B A$. Another useful matrix identity is the following $\left(A^{-1}+B^{\prime} C^{-1} B\right)^{-1} B^{\prime} C^{-1}=A B^{\prime}\left(B A B^{\prime}+\right.$ $C)^{-1}$. 
From this inequality, we further conclude that by picking $\epsilon>0$ such that

$$
r:=(1+\epsilon) e^{-\gamma \max _{j} \tau_{D}}<1
$$

it follows that $V$ is bounded and $V\left(t_{k}\right) \rightarrow \frac{1}{1-r}\left(\frac{1}{\epsilon} \max _{j} a_{j}+\right.$ $\left.\max _{j} b_{j}\right) e^{\gamma \max _{j}\left\{N_{0} \tau_{D}\right\}}$ as $k \rightarrow \infty$. Since for every finite time, $P$ is positive definite, $V$ must be finite on any finite interval and therefore so must be $\tilde{x}$ and $\hat{x}$. Global existence of solution follows. It is also straightforward to conclude from (64) that the ISS-like bound (42) holds.

Proof: [Lemma 2] From (25), and (27), we conclude that $P(t)$ can be written as

$$
\begin{array}{r}
P(t)=\Psi(0, t)^{\prime} P_{0} \Psi(0, t) \\
+\int_{0}^{t} \Psi(\tau, t)^{\prime} P(\tau) G G^{\prime} P(\tau) \Psi(\tau, t) d \tau \\
+\sum_{i=1}^{k} \Psi\left(t_{i}, t\right)^{\prime} W\left(t_{i}\right) \Psi\left(t_{i}, t\right)
\end{array}
$$

where for any $\tau \in\left[t_{i}, t_{i+1}\right)$ and $\sigma \in\left[t_{j}, t_{j+1}\right)$

$$
\Psi(\tau, \sigma):=\left\{\begin{array}{cc}
\Psi_{i}(\tau, \sigma), & i=j \\
\Psi_{i}\left(\tau, t_{i+1}\right) \Psi_{i+1}\left(t_{i+1}, t_{i+2}\right) \cdots \Psi_{j}\left(t_{j}, \sigma\right), & i<j
\end{array}\right.
$$

and $\Psi_{i}(t, \tau)$ denotes the state transition matrix of $\dot{z}=(A+$ $\left.G G^{\prime} P\right) z$ for $t_{i} \leq \tau \leq t<t_{i+1}$. We now show that

$$
P(t) \geq \mu I>0 \quad \forall t \geq 0
$$

for some positive constant $\mu$. Let $x \in \mathbb{R}^{n}$ be an arbitrary vector. Using (65) it follows that

$$
\begin{aligned}
x^{\prime} P(t) x \geq \int_{0}^{t} z(\tau)^{\prime} P(\tau) G G^{\prime} P(\tau) & z(\tau) d \tau \\
& +\sum_{i=1}^{k} z\left(t_{i}\right)^{\prime} W\left(t_{i}\right) z\left(t_{i}\right)
\end{aligned}
$$

where $z(\tau):=\Psi(\tau, t) x$, and satisfies

$$
\frac{d}{d \tau} z(\tau, t)=\left(A+G G^{\prime} P\right) z(\tau) \quad 0 \leq \tau \leq t .
$$

Since $P_{0}>0$, then for $t \geq t_{k-\mathbf{N}}$, we conclude that

$$
x^{\prime} P(t) x \geq \alpha(t)^{2}+\beta(t)^{2}
$$

where $\alpha(t):=\left(\sum_{i=k-\mathbf{N}}^{k}\left\|W\left(t_{i}\right)^{\frac{1}{2}} z\left(t_{i}\right)\right\|^{2}\right)^{\frac{1}{2}}, \beta(t):=$ $\left(\int_{t-t_{k-\mathbf{N}}}^{t}\left\|G^{\prime} P(\tau) z(\tau)\right\|^{2} d \tau\right)^{\frac{1}{2}}$. We now proceed to compute a lower-bound for $x^{\prime} P(t) x$ by computing a lower-bound for the right-hand-side of (68). Fix some $t \geq t_{k-\mathbf{N}}$. Applying the variation of constants formula to (67), we get

$z(\tau)=\Phi(\tau, t) x+\int_{t}^{\tau} \Phi(\tau, \sigma) G G^{\prime} P(\sigma) z(\sigma) d \sigma \quad 0 \leq \tau \leq t$.

Therefore,

$$
\begin{aligned}
& \left(\sum_{i=k-\mathbf{N}}^{k}\left\|W\left(t_{i}\right)^{\frac{1}{2}} \Phi\left(t_{i}, t\right) x\right\|^{2}\right)^{\frac{1}{2}} \leq \alpha \\
& +\left(\sum_{i=k-\mathbf{N}}^{k}\left\|W\left(t_{i}\right)^{\frac{1}{2}} \int_{t}^{t_{i}} \Phi\left(t_{i}, \sigma\right) G G^{\prime} P(\sigma) z(\sigma) d \sigma\right\|^{2}\right)^{\frac{1}{2}}
\end{aligned}
$$

Moreover, using the Schwartz inequality, we conclude that

$$
\begin{aligned}
& \sum_{i=k-\mathbf{N}}^{k}\left\|W\left(t_{i}\right)^{\frac{1}{2}} \int_{t}^{t_{i}} \Phi\left(t_{i}, \sigma\right) G G^{\prime} P(\sigma) z(\sigma) d \sigma\right\|^{2} \leq \\
& \sum_{i=k-\mathbf{N}}^{k} \int_{t_{i}}^{t}\left\|W\left(t_{i}\right)^{\frac{1}{2}} \Phi\left(t_{i}, \sigma\right) G\right\|^{2} d \sigma \int_{t_{i}}^{t}\left\|G^{\prime} P(\sigma) z(\sigma)\right\|^{2} d \sigma
\end{aligned}
$$

and because $\left\|W\left(t_{i}\right)^{\frac{1}{2}} \Phi\left(t_{i}, \sigma\right) G\right\|$ is uniformly bounded for $\sigma \in\left[t_{i}, t\right]$, we further conclude that the right-hand-side term is bounded by

$$
\sum_{i=k-\mathbf{N}}^{k} c \int_{t_{i}}^{t}\left\|G^{\prime} P(\sigma) z(\sigma)\right\|^{2} d \sigma \leq c \mathbf{N} \beta(t)^{2}
$$

for an appropriately defined constant $c$. From (69) and (70), it then follows that

$$
\left(\sum_{i=k-\mathbf{N}}^{k}\left\|W\left(t_{i}\right)^{\frac{1}{2}} \Phi\left(t_{i}, t\right) x\right\|^{2}\right)^{\frac{1}{2}} \leq \alpha(t)+\sqrt{c \mathbf{N}} \beta(t)
$$

$\forall t \geq t_{k-\mathbf{N}}$. From this, (68), and assuming without of generality that $c \mathbf{N} \geq 1$, it is straightforward to conclude that

$$
\begin{aligned}
x^{\prime} P(t) x & \geq \alpha^{2}+\beta^{2} \geq \frac{(\alpha+\sqrt{c \mathbf{N}} \beta)^{2}}{2 c \mathbf{N}} \\
& \geq \frac{1}{2 c \mathbf{N}} \sum_{i=k-\mathbf{N}}^{k}\left\|W\left(t_{i}\right)^{\frac{1}{2}} \Phi\left(t_{i}, t\right) x\right\|^{2} .
\end{aligned}
$$

Performing the change of coordinates $j=i-k+N$, yields

$$
\begin{aligned}
& x^{\prime} P(t) x \\
& \geq \frac{1}{2 c \mathbf{N}} \sum_{j=0}^{\mathbf{N}} x^{\prime} \Phi\left(t_{j+k-\mathbf{N}}, t\right)^{\prime} W\left(t_{j+k-\mathbf{N}}\right) \Phi\left(t_{j+k-\mathbf{N}}, t\right) x \\
& =\frac{1}{2 c \mathbf{N}} x^{\prime} \Phi\left(t_{k-\mathbf{N}}, t\right) \times \\
& \left(\sum_{j=0}^{\mathbf{N}} \Phi\left(t_{j+k-\mathbf{N}}, t_{k-\mathbf{N}}\right)^{\prime} W\left(t_{j+k-\mathbf{N}}\right) \Phi\left(t_{j+k-\mathbf{N}}, t_{k-\mathbf{N}}\right)\right) \times \\
& \quad \Phi\left(t_{k-\mathbf{N}}, t\right) x .
\end{aligned}
$$

From this and (43), we finally obtain

$$
x^{\prime} P(t) x \geq \frac{\epsilon}{2 c}\left\|\Phi\left(t_{k-\mathbf{N}}, t\right) x\right\|^{2} \geq \frac{\epsilon}{2 c\left\|\Phi\left(t, t_{k-\mathbf{N}}\right)\right\|^{2}}\|x\|^{2}
$$

$\forall t \geq t_{k-\mathbf{N}}$. This proves that (66) holds with $\mu$ equal to the smallest of $\frac{\epsilon}{2 c\left\|\Phi\left(t, t_{k-\mathbf{N}}\right)\right\|^{2}}$ and the smallest eigenvalue of $P(t)$ on the closed interval $\left[0, t_{k-\mathbf{N}}\right]$. The latter is strictly positive since $P(t)$ is positive definite for any finite time $t$.

\section{REFERENCES}

[1] B. K. Ghosh, M. Jankovic, and Y. T. Wu, "Perspective problems in system theory and its application in machine vision," J. Mathematical Syst. Estimation Contr., vol. 4, no. 1, pp. 3-38, 1994.

[2] B. K. Ghosh and E. P. Loucks, "A perspective theory for motion and shape estimation in machine vision," SIAM J. Contr. Optimization, vol. 33, no. 5, pp. 1530-1559, 1995.

[3] S. Takahashi and B. K. Ghosh, "Motion and shape parameters identification with vision and range," in Proc. of the 2001 Amer. Contr. Conf., vol. 6, June 2001, pp. 4626-4631. 
[4] W. P. Dayawansa, B. K. Ghosh, C. Martin, and X. Wang, "A necessary and sufficient condition for the perspective observability problem," Syst. \& Contr. Lett., vol. 25, pp. 159-166, 1995.

[5] H. Inaba, A. Yoshida, R. Abdursul, and B. K. Ghosh, "Observability of perspective dynamical systems," in Proc. of the 39th Conf. on Decision and Contr., vol. 5, 2000, pp. 5157-5162.

[6] E. D. Sontag, Mathematical Control Theory: Deterministic Finite Dimensional Systems, 2nd ed. Springer Verlag, 1998.

[7] R. E. Mortensen, "Maximum likelihood recursive nonlinear filtering," J. Opt. Theory and Applications, vol. 2, pp. 386-394, 1968.

[8] O. J. Hijab, "Minimum energy estimation," Ph.D. dissertation, University of California, Berkeley, 1980.

[9] W. M. McEneaney, "Robust/H-infinity filtering for nonlinear systems," Syst. \& Contr. Lett., no. 33, pp. 315-325, 1998.

[10] A. J. Krener, "The convergence of the minimum energy estimator," in New Trends in Nonlinear Dynamics and Control, and Their Applications, ser. Lecture Notes in Control and Information Sciences, W. Kang, Ed. Springer-Verlag, 2003.

[11] W. Zhang, M. Branicky, and S. Phillips, "Stability of networked control systems," IEEE Contr. Syst. Mag., vol. 21, no. 1, pp. 84-99, Feb. 2001.

[12] L. Matthies, T. Kanade, and R. Szeliski, "Kalman filter-based algorithms for estimating depth from image sequences," Int. J. of Comput. Vision, vol. 3, pp. 209-236, 1989.

[13] M. Jankovic and Ghosh, "Visually guided ranging from observations of points, lines and curves via an identifier based nonlinear observer," Syst. \& Contr. Lett., vol. 25, pp. 63-73, 1995.

[14] S. Soatto, R. Frezza, and P. Perona, "Motion estimation via dynamic vision," IEEE Trans. on Automat. Contr., vol. 41, no. 3, pp. 393-413, Mar. 1996.

[15] I. Kaminer, A. M. Pascoal, W. Kang, and O. Yakimenko, "Integrated vision/inertial navigation systems design using nonlinear filtering," IEEE Trans. Aerospace and Electronic Syst., vol. 37, no. 1, pp. 158-172, Jan. 2001.

[16] A. Chiuso, P. Favaro, H. Jin, and S. Soatto, "Structure from motion causally integrated over time," IEEE Trans. on Pattern Anal. Mach. Intell., vol. 24, no. 4, pp. 523-535, Apr. 2002.

[17] H. Rehbinder and B. K. Ghosh, "Pose estimation using line-based dynamic vision and inertial sensors," IEEE Trans. on Automat. Contr., vol. 48, no. 2, pp. 186-199, Feb. 2003.

[18] Y. Ma, J. Kosecka, and S. Sastry, "Vision guided navigation for a nonholonomic mobile robot," IEEE Trans. Robot. Automat., vol. 15, no. 3, pp. 521-536, 1999

[19] O. Shakernia, Y. Ma, T. Koo, and S. Sastry, "Landing an unmanned air vehicle: vision based motion estimation and nonlinear control," Asian J. Contr., vol. 1, no. 3, pp. 128-145, 1999.

[20] J. Hespanha, O. Yakimenko, I. Kaminer, and A. Pascoal, "Linear parametrically varying systems with brief instabilities: an application to integrated vision/IMU navigation," in Proc. of the 40th Conf. on Decision and Contr., Dec. 2001.

[21] J. P. Hespanha, "State estimation and control for systems with perspective outputs," in Proc. of the 41st Conf. on Decision and Contr., Dec. 2002.

[22] A. P. Aguiar and J. P. Hespanha, "Pose estimation of autonomous vehicles using visual information: A minimum-energy estimator approach," in Proc. of IAV2004 - 5th IFAC/EURON Symp. on Intel. Auton. Vehicles, July 2004.

[23] — "State estimation for continuous-time systems with perspective outputs from discrete noisy time-delayed measurements," in Proc. of the 43rd Conf. on Decision and Contr., Dec. 2004.

[24] M. James, J. Baras, and R. Elliott, "Output feedback risk-sensitive control and differential games for continuous-time nonlinear systems," in Proc. of the 32nd Conf. on Decision and Contr., San Antonio, TX, USA, 1993, pp. 3357-3360.

[25] — , "Risk-sensitive control and dynamic games for partially observed discrete-time nonlinear systems," IEEE Trans. on Automat. Contr. vol. 39, no. 4, p. 780792, 1994.

[26] P. J. Antsaklis and A. N. Michel, Linear Systems, ser. McGraw-Hill Series in Electrical and Computer Engineering. New York: McGrawHill, 1997.

[27] E. Sontag, "Smooth stabilization implies coprime factorization," IEEE Trans. on Automat. Contr., vol. 34, no. 4, pp. 435-443, 1989.

[28] E. Sontag and A. Teel, "Changing supply functions in input/state stable systems," IEEE Trans. on Automat. Contr., vol. 40, no. 8, pp. 1476-1478, 1995.

[29] H. K. Khalil, Nonlinear Systems, 2nd ed. New Jersey, USA: PrenticeHall, 1996.
[30] E. Panteley, A. Loria, and A. Teel, "Relaxed persistency of excitation for uniform asymptotic stability," IEEE Trans. on Automat. Contr., vol. 46, no. 12, pp. 1874 - 1886, Dec. 2001.

[31] J. P. Hespanha and A. S. Morse, "Stability of switched systems with average dwell-time," in Proc. of the 38th Conf. on Decision and Contr., Dec. 1999.

[32] Y. Ma, J. Kosecka, S. Soatto, and S. Sastry, An Invitation to 3-D Vision. Springer Verlag, 2003.

[33] R. A. Horn and C. R. Johnson, Topics in Matrix Analysis. Cambridge: Cambridge University Press., 1994.

[34] A. P. Aguiar and J. P. Hespanha, "Minimum-energy state estimation for systems with perspective outputs and state constraints," in Proc. of the 42nd Conf. on Decision and Contr., Dec. 2003.

[35] ActivMedia. [Online]. Available: http://www.activmedia.com/

[36] M. Aicardi, G. Casalino, A. Bicchi, and A. Balestrino, "Closed loop steering of unicycle-like vehicles via Lyapunov techniques," IEEE Rob. \& Autom. Mag., vol. 2, no. 1, pp. 27-35, 1995.

[37] B. Kim and P. Tsiotras, "Controllers for unicycle-type wheeled robots: Theoretical results and experimental validation," IEEE Trans. Robot. Automat., vol. 18, no. 3, pp. 294-307, 2002.

[38] A. Naylor and G. Sell, "Linear operator theory in engineering and science," Applied Mathematical Sciences. Springer-Verlag, no. 40, New York, 1982.

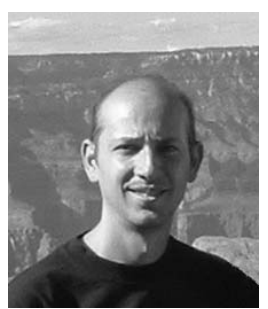

A. Pedro Aguiar received the Licenciatura, M.S. and Ph.D. in electrical and computer engineering from the Instituto Superior Técnico, Technical University of Lisbon, Portugal in 1994, 1998 and 2002, respectively. From 2002 to 2005, he was a post-doctoral researcher at the Center for Control, Dynamical-Systems, and Computation at the University of California, Santa Barbara. Currently, Dr. Aguiar holds a Senior Research position with the Institute for Systems and Robotics, Instituto Superior Técnico.

His research interests include modeling, control, navigation, and guidance of autonomous vehicles; nonlinear control; switched and hybrid systems; tracking, path-following; performance limitations; nonlinear observers; the integration of machine vision with feedback control; and coordinated/cooperative control of a group of autonomous vehicles.

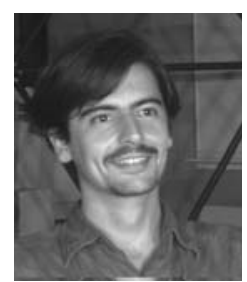

João P. Hespanha received the Licenciatura in electrical and computer engineering from the Instituto Superior Técnico, Lisbon, Portugal in 1991 and the M.S. and Ph.D. degrees in electrical engineering and applied science from Yale University, New Haven, Connecticut in 1994 and 1998, respectively. He currently holds an Associate Professor position with the Department of Electrical and Computer Engineering, the University of California, Santa Barbara. From 1999 to 2001, he was an Assistant Professor at the University of Southern California, Los Angeles. Dr. Hespanha is the associate director for the Center for Control, Dynamicalsystems, and Computation (CCDC) and an executive committee member for the Institute for Collaborative Biotechnologies (ICB), an Army sponsored University Affiliated Research Center (UARC).

His research interests include hybrid and switched systems; the modeling and control of communication networks; distributed control over communication networks (also known as networked control systems); the use of vision in feedback control; stochastic modeling in biology; and the control of haptic devices. He is the author of over one hundred technical papers and the PI and co-PI in several federally funded projects.

Dr. Hespanha is the recipient of the Yale Universitys Henry Prentiss Becton Graduate Prize for exceptional achievement in research in Engineering and Applied Science, a National Science Foundation CAREER Award, the 2005 Automatica Theory/Methodology best paper prize, and the best paper award at the 2nd Int. Conf. on Intelligent Sensing and Information Processing. Since 2003, he has been an Associate Editor of the IEEE Transactions on Automatic Control.

More information about Dr. Hespanha's research can be found at http://www.ece.ucsb.edu/ hespanha 